\title{
Total Synthesis of $(+)$-Acutiphycin
}

\author{
Supporting Information \\ Ryan M. Moslin, Timothy, F. Jamison* \\ Massachusetts Institute of Technology, Department of Chemistry, Cambridge, Massachusetts 02139 \\ TABLE OF CONTENTS
}

Procedures and spectral data

References

Spectra page $\mathrm{S} 2-\mathrm{S} 36$

page $\mathrm{S} 37$

page $\mathrm{S} 38-\mathrm{S} 111$ 


\section{Supporting Information}

\section{General Methods}

Unless otherwise noted, all reactions were performed under an oxygen-free atmosphere of argon using standard Schlenk-line techniques. Diisopropylamine was distilled from calcium hydride and stored over potassium hydroxide. Tetrahydrofuran (THF) and diethyl ether were freshly distilled over sodium/benzophenone ketyl. Dichloromethane (DCM), xylenes, and toluene were freshly distilled from calcium hydride. Where noted reagents were azeotroped with toluene by concentrating them on a rotoevaporator, which was backfilled with argon. Ethoxyethyne was received as a red solution in hexanes from GFS, it was distilled under argon

and collected as a clear oil, ${ }^{1} \mathrm{H}$ NMR was used to determine its weight $\%$ in hexanes and it could be stored for up to a month under argon at $-4{ }^{\circ} \mathrm{C}$. All other reagents were used as received unless otherwise noted.

${ }^{1} \mathrm{H}$ NMR was performed on a $500 \mathrm{MHz}$ inverse probe instrument, ${ }^{13} \mathrm{C} \mathrm{NMR}$ was performed on a $500 \mathrm{MHz}$ broadband probe instrument. Deuterochloroform $\left(\mathrm{CDCl}_{3}\right)$, which had been filtered through activated basic alumina prior to use, was used as the solvent. Unless otherwise noted the reference peak was set to $\delta 7.27$ and $\delta 77.23$ ppm from tetramethylsilane for the ${ }^{1} \mathrm{H}$ and ${ }^{13} \mathrm{C}$ spectra respectively. Infrared (IR) spectra were recorded as a thin film between $\mathrm{NaCl}$ plates on a FT-IR System transform spectrometer. High resolution mass spectra (HRMS) were obtained on a Fourier transform mass spectrometer. 
(+)-5-Benzyloxy-3-(tert-butyl-diphenyl-silanyloxy)-methyl pentanoate (50):

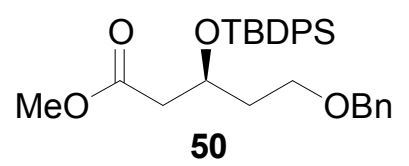

To a cold $\left(0^{\circ} \mathrm{C}\right)$ solution of $7^{1}(8.74 \mathrm{~g}, 37 \mathrm{mmol})$ in DMF $(50 \mathrm{~mL})$ was added imidazole (4.9 g, $72 \mathrm{mmol}$ ) and chloro-tert-butyl-diphenylsilane $(11.4 \mathrm{ml}, 44 \mathrm{mmol})$, the mixture was warmed to room temperature and stirred overnight. The reaction was quenched with water and then extracted with diethyl ether. The combined organic extracts were washed with water $(2 \mathrm{x})$ and brine, then dried over magnesium sulfate, filtered, concentrated and purified by silica gel chromatography (50:1 hexanes/diethyl ether $\rightarrow 6: 1$ hexanes/ethyl acetate) to give a clear oil. The oil was placed in a large sublimation apparatus and heated to $70{ }^{\circ} \mathrm{C}$ under vacuum $(0.01$ $\mathrm{mmHg}$ ) for 24 hours resulting in the collection of an unidentified white solid, recovery of the residual oil gave $50(15.7 \mathrm{~g}, 90 \%)$. $[\alpha]_{\mathrm{D}}+16.9\left(c 1.0,22{ }^{\circ} \mathrm{C}, \mathrm{CHCl}_{3}\right)$; chiral HPLC analysis (OD column, 99:1 hexane:isopropanol $0.7 \mathrm{ml} / \mathrm{min}) \mathrm{R}_{\mathrm{F}}(S)=9.70 \mathrm{~min}, \mathrm{R}_{\mathrm{F}}(R)=10.78 \mathrm{~min}$; IR 3071 (m), 2932 (s), 2858 (s), 1741 (s), 1473 (s), 1428 (s), 1362(s), 1169 (s); ${ }^{1} \mathrm{H}$ NMR (500 MHz, $\left.\mathrm{CDCl}_{3}\right) \delta 7.68(\mathrm{~m}, 4 \mathrm{H}), 7.40(\mathrm{~m}, 4 \mathrm{H}), 7.30(\mathrm{~m}, 4 \mathrm{H}), 4.37$ (quint, $\left.J=6.0 \mathrm{~Hz}, 1 \mathrm{H}\right), 4.33(\mathrm{~s}, 2 \mathrm{H})$, $3.53(\mathrm{~s}, 3 \mathrm{H}), 3.49(\mathrm{~m}, 1 \mathrm{H}), 2.53(\mathrm{~d}, J=6.0 \mathrm{~Hz}, 2 \mathrm{H}), 1.84(\mathrm{~m}, 2 \mathrm{H}), 1.03(\mathrm{~s}, 9 \mathrm{H}) ;{ }^{13} \mathrm{C}(125.8 \mathrm{MHz}$ $\left.\mathrm{CDCl}_{3}\right) \delta 172.0,138.5,136.1,136.1,134.0,129.8,129.8,128.5,127.8,127.7,127.7,127.6,72.9$ 68.5, 66.7, 51.6, 42.4, 37.1, 27.1, 19.5; HRMS $m / z$ (ESI, $\mathrm{M}+\mathrm{Na}^{+}$) calcd 499.2275, found 499.2257.

(+)-methyl 3-(tert-butyldiphenylsilyloxy)-5-oxopentanoate (3): 


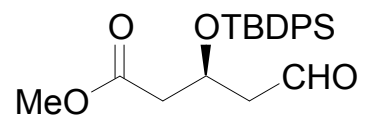

3

50 (450 mg, $0.95 \mathrm{mmol})$ was dissolved in reagent grade methanol $(20 \mathrm{~mL})$ and placed in a high pressure apparatus along with $200 \mathrm{mg}$ of palladium on carbon $(10 \%$ by weight, $50 \%$ water). The vessel was placed under vacuum $(20 \mathrm{mmHg})$ and backfilled with $\mathrm{H}_{2}$, this cycle was repeated twice, and the vessel was charged to 40 psi with $\mathrm{H}_{2}$ and stirred overnight. The reaction solution was filtered through celite, eluting with ethyl acetate, and concentrated to give the primary alcohol as a clear oil. The oil was dissolved in DCM $(1.8 \mathrm{~mL})$ and added dropwise to a solution of Dess-Martin periodinane $(806 \mathrm{mg}, 1.9 \mathrm{mmol})$ and pyridine $(338 \mu \mathrm{l}, 4.2 \mathrm{mmol})$ in $\operatorname{DCM}(9 \mathrm{ml})$ at room temperature. After stirring for $1.5 \mathrm{~h}, 24 \mathrm{~mL}$ of a 1:1 solution of saturated aqueous sodium bicarbonate and sodium bisulfite was added and the biphasic solution was stirred until both phases were clear. The layers were separated and the aqueous layer extracted with diethyl ether. The combined organic layers were washed with saturated aqueous sodium bicarbonate and brine, dried over magnesium sulfate, filtered, concentrated, and purified by chromatography $\left(2: 1\right.$ hexanes/diethyl ether) to give $304 \mathrm{mg}(84 \%)$ of $\mathbf{3}$ as a clear oil. $[\alpha]_{\mathrm{D}}+0.5$ (c 1.6, $21^{\circ} \mathrm{C}, \mathrm{CHCl}_{3}$ ); IR 2895 (s), 2933 (s), 2859 (s), 2361 (m), 1733 (s), 1112 (s), 704 (s); ${ }^{1} \mathrm{H}$ NMR (500 MHz, $\left.\mathrm{CDCl}_{3}\right) \delta 9.63(\mathrm{t}, J=2.5 \mathrm{~Hz}, 1 \mathrm{H}), 7.67(\mathrm{~m}, 4 \mathrm{H}), 7.46(\mathrm{~m}, 2 \mathrm{H}), 7.40(\mathrm{~m}, 4 \mathrm{H})$, 4.62 (quint, $J=6.0 \mathrm{~Hz}, 1 \mathrm{H}), 3.58(\mathrm{~s}, 3 \mathrm{H}), 2.69(\mathrm{ddd}, J=16.5,6.5,2.0 \mathrm{~Hz}, 1 \mathrm{H}), 2.58(\mathrm{~m}, 3 \mathrm{H})$, $1.04(\mathrm{~s}, 9 \mathrm{H}) ;{ }^{13} \mathrm{C}\left(125.8 \mathrm{MHz}, \mathrm{CDCl}_{3}\right) \delta 201.0,171.2,136.0,136.0,133.4,133.2,130.2,130.1$, 128.0, 127.9, 66.1, 51.8, 50.6, 42.0, 27.0, 19.4; HRMS $m / z\left(E S I, M+\mathrm{Na}^{+}\right)$calcd 407.1649, found 407.1638 .

(+)-1-(tert-Butyl-dimethyl-silanyloxy)-2,4,4-trimethyl-hex-5-en-3-ol (9): 


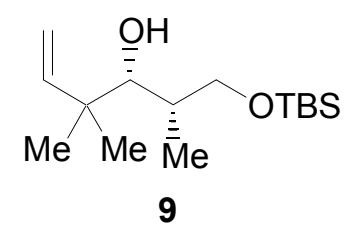

To a cold $\left(0{ }^{\circ} \mathrm{C}\right)$ suspension of indium $(4.82 \mathrm{~g}, 41.99 \mathrm{mmole})$ in DMF $(38 \mathrm{~mL})$ a solution of aldehyde $\mathbf{8}^{2}$ (3.85 g, 19.1 mmole) and prenyl bromide (6.6 mL, 57.3 mmole) in DMF (14 mL) were added in a dropwise fashion. After warming to room temperature and stirring overnight the reaction was quenched via the addition of $0.5 \mathrm{M} \mathrm{HCl}(30 \mathrm{~mL})$, the solution was diluted with diethyl ether, the two layers were separated and the aqueous layer extracted with diethyl ether. The combined organics were washed with water and brine, dried over magnesium sulfate, concentrated and crudely purified by chromatography (10:1 hexanes:diethyl ether) to obtain 9 as a mixture of diastereomers $(3.47 \mathrm{~g}, 83: 17$ determined by GC). This material was then resubjected to chromatography (25:1 hexanes:diethyl ether) to give $\mathbf{9}$ as a $96: 4$ mixture of diastereomers $(2.74 \mathrm{~g},>99 \%$ ee, $52 \%$ yield $)$. Data is for the syn isomer only. $[\alpha]_{\mathrm{D}}+13.9(c$ $0.79,22^{\circ} \mathrm{C}, \mathrm{CHCl}_{3}$ ); chiral $\mathrm{GC}$ analysis (BDA column, $\left.120{ }^{\circ} \mathrm{C}, 1.6 \mathrm{~mL} / \mathrm{min}_{2}\right) \mathrm{R}_{\mathrm{F}}(R, S)=20.8$ $\min , \mathrm{R}_{\mathrm{F}}(R, R)=21.8 \min , \mathrm{R}_{\mathrm{F}}(S, R)=18.7 \min , \mathrm{R}_{\mathrm{F}}(S, S)=18.3 \min ; \mathrm{IR} 3510(\mathrm{bm}), 2957(\mathrm{~s})$, 2930 (s), 2859 (s), 1636 (w), 1472 (m), 1390 (m), 1256 (s), 1093 (s), 1006 (m), 910 (m), 837 (s), $776(\mathrm{~s}) ;{ }^{1} \mathrm{H}$ NMR $\left(500 \mathrm{MHz}, \mathrm{CDCl}_{3}\right) \delta 5.98(\mathrm{dd}, J=18.0,10.5 \mathrm{~Hz}, 1 \mathrm{H}), 5.01(\mathrm{dd}, J=18.0,1.5$ $\mathrm{Hz}, 1 \mathrm{H}), 5.01(\mathrm{dd}, J=10.5,1.5 \mathrm{~Hz}, 1 \mathrm{H}), 3.63(\mathrm{dd}, J=9.5,4.0 \mathrm{~Hz}, 1 \mathrm{H}), 3.56(\mathrm{dd}, J=9.5,5.0 \mathrm{~Hz}$, 2H), $2.52(\mathrm{~d}, J=3.0 \mathrm{~Hz}, 1 \mathrm{H}), 1.86(\mathrm{~m}, 1 \mathrm{H}), 1.07(\mathrm{~s}, 3 \mathrm{H}), 1.05(\mathrm{~s}, 3 \mathrm{H}), 0.92(\mathrm{~d}, J=7.0 \mathrm{~Hz}, 3 \mathrm{H})$, 0.90 (s, 9H), $0.06(\mathrm{~s}, 6 \mathrm{H}) ;{ }^{13} \mathrm{C}\left(125.8 \mathrm{MHz}, \mathrm{CDCl}_{3}\right) \delta 146.2,111.9,80.1,70.4,41.8,35.8,26.1$, 24.9, 24.2, 18.4, 11.2, -5.3, -5.4; HRMS $m / z\left(E S I, M+\mathrm{Na}^{+}\right)$calcd 295.2064, found 295.2060.

\section{(+)-3-Hydroxy-2,4,4-trimethyl-hex-5-enal (10):}




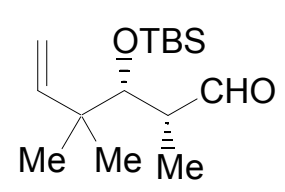

10

2,6-Lutidine (3.49 mL, 30.0 mmole) was added to a solution of 9 (2.72 g, 10.0 mmole) in $\operatorname{DCM}(60 \mathrm{~mL})$ and the mixture was cooled to $-78{ }^{\circ} \mathrm{C}$. tert-Butyldimethylsilyltriflate (TBSOTf) (3.45 mL, 15.0 mmole) was added to the cooled solution, which was warmed to $0{ }^{\circ} \mathrm{C}$, stirred for 1 hour then quenched with water. The two phases were separated and the aqueous layer extracted with diethyl ether, the combined organics were washed with water and brine, dried over magnesium sulfate, filtered, concentrated and purified by silica gel chromatography (hexanes) to give $3.42 \mathrm{~g}(88 \%)$ of the bis-silyl-ether. A portion of this material $(3.18 \mathrm{~g}, 8.22$ mmole) was dissolved in methanol $(220 \mathrm{~mL})$ and DCM $(156 \mathrm{~mL})$ and cooled to $0{ }^{\circ} \mathrm{C}$. A solution of CSA (450 mg, 1.94 mmole) in methanol $(63 \mathrm{~mL})$ was added and the solution stirred for 4 hours at $0{ }^{\circ} \mathrm{C}$ then quenched with saturated aqueous sodium bicarbonate and extracted with ethyl acetate. The combined organic layers were washed with $1 \mathrm{M} \mathrm{NaOH}$ and brine, dried over magnesium sulfate, concentrated and purified by silica gel chromatography to give $2.08 \mathrm{~g}(93 \%)$ of the primary alcohol. To a cold $\left(0^{\circ} \mathrm{C}\right)$ solution of TPAP $(140 \mathrm{mg}, 0.40 \mathrm{mmole}), \mathrm{NMO}(2.13 \mathrm{~g}$, $12 \mathrm{mmole}$ ), and powdered $4 \AA$ molecular sieves (c. $100 \mathrm{mg}$ ) in DCM (40 mL) was added a solution of the above alcohol $(1.09 \mathrm{~g}, 4.0 \mathrm{mmole})$ in $16 \mathrm{~mL}$ DCM. The reaction was stirred for 1.5 hours then passed through a silica pad (eluting with ethyl acetate). The eluent was concentrated purified by chromatography (6:1 hexanes:diethyl ether) to give $960 \mathrm{mg}(89 \%)$ of 10. $[\alpha]_{\mathrm{D}}+17.5\left(\mathrm{c} 20.57,22{ }^{\circ} \mathrm{C}, \mathrm{CHCl}_{3}\right)$; IR 3084 (bm), 2958 (s), 2931 (s), 2859 (s), 1726 (s), 1638 (w), 1473 (s), 1381 (m), 1253 (s), 1142 (m), 1108 (s), 1046 (s), 915 (s), 838 (s), 775 (s), $670(\mathrm{~m}) ;{ }^{1} \mathrm{H} \mathrm{NMR}\left(500 \mathrm{MHz}, \mathrm{CDCl}_{3}\right) \delta 9.64(\mathrm{~s}, 1 \mathrm{H}), 5.90(\mathrm{dd}, J=17.0,11.5 \mathrm{~Hz}, 1 \mathrm{H}), 5.02(\mathrm{dd}$, $J=11.5,1.5 \mathrm{~Hz}, 1 \mathrm{H}), 5.01(\mathrm{dd}, J=17.0,1.5 \mathrm{~Hz}, 1 \mathrm{H}), 4.04(\mathrm{~d}, J=2.0 \mathrm{~Hz}, 1 \mathrm{H}), 2.50\left(\mathrm{dq}, J_{\mathrm{q}}=\right.$ 
7.5, $\left.J_{\mathrm{d}}=2.0 \mathrm{~Hz}, 1 \mathrm{H}\right), 1.15(\mathrm{~d}, J=7.0 \mathrm{~Hz}, 3 \mathrm{H}), 1.04(\mathrm{~s}, 3 \mathrm{H}), 1.03(\mathrm{~s}, 3 \mathrm{H}), 0.91(\mathrm{~s}, 9 \mathrm{H}), 0.09(\mathrm{~s}$, $3 \mathrm{H}),-0.08(\mathrm{~s}, 3 \mathrm{H}) ;{ }^{13} \mathrm{C}\left(125.8 \mathrm{MHz}, \mathrm{CDCl}_{3}\right) \delta 205.6,145.6,112.6,75.5,49.2,43.2,26.3,25.5$, 23.1, 18.7, 9.6, -3.4, -4.3; HRMS $m / z\left(\mathrm{ESI}, \mathrm{M}+\mathrm{Na}^{+}\right.$) calcd 293.1907, found 293.1907.

\section{(+)-syn-1,6-enyne (5):}

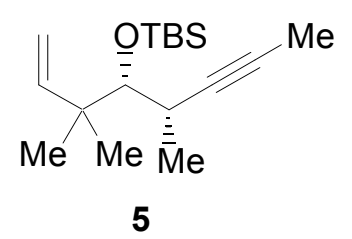

Potassium tert-butoxide (678 $\mathrm{mg}, 6.04 \mathrm{mmole})$ was dissolved in THF (39 $\mathrm{mL})$ and then cooled to $-78{ }^{\circ} \mathrm{C}$, dimethylphosphonodiazomethane ( $\left.720 \mathrm{mg}, 6.04 \mathrm{mmole}\right)$ was added in THF $(13 \mathrm{~mL})$ dropwise over 15 minutes. After stirring for 15 minutes a solution of $\mathbf{1 0}(1.02 \mathrm{~g}, 3.78$ mmole) in THF $(13 \mathrm{~mL})$ was added dropwise over 20 minutes. The reaction was stirred at -78 ${ }^{\circ} \mathrm{C}$ for 4 hours, then warmed to room temperature and stirred for an additional 1.5 hours, quenched with water and extracted with diethyl ether. The combined organic extracts were washed with brine, dried over magnesium sulfate, concentrated and purified by silica gel chromatography (hexanes) to give $875 \mathrm{mg}(86 \%)$ of the terminal alkyne, a portion of which was used in the subsequent step. $n$-BuLi $(2.5 \mathrm{M}$ in hexanes, $6.14 \mathrm{~mL}, 15.3 \mathrm{mmol})$ was added dropwise to a cold $\left(-10^{\circ} \mathrm{C}\right)$ solution of $i \operatorname{Pr}_{2} \mathrm{NH}(2.22 \mathrm{~mL}, 15.8 \mathrm{mmol})$ in THF $(51 \mathrm{~mL})$ the solution was stirred for 15 minutes, then cooled to $-78{ }^{\circ} \mathrm{C}$. The terminal alkyne ( $860 \mathrm{mg}, 3.2$ mmol), DMPU (Aldrich, $3.2 \mathrm{~mL}, 26.9 \mathrm{mmol}$ ), and MeI (filtered through activated basic alumina, $0.956 \mathrm{~mL}, 15.4 \mathrm{mmol})$ were added to the solution at $-78^{\circ} \mathrm{C}$, the solution was warmed to $0{ }^{\circ} \mathrm{C}(5$ minutes) then to room temperature $(2.5 \mathrm{~h})$. The reaction was quenched via the addition of water, and the product extracted with diethyl ether. The combined organic layers were washed with brine, dried over magnesium sulfate, filtered, concentrated and purified by chromatography (hexanes) to give $5(840 \mathrm{mg}, 94 \%) .[\alpha]_{\mathrm{D}}+3.1\left(c 11.78,21{ }^{\circ} \mathrm{C}, \mathrm{CHCl}_{3}\right)$; IR $3083(\mathrm{w}), 2959(\mathrm{~s})$, 
2930 (s), $2858(\mathrm{~s}), 1638(\mathrm{w}), 1473(\mathrm{~m}), 1416(\mathrm{~m}), 1360(\mathrm{~m}), 1252(\mathrm{~s}), 1110(\mathrm{~s}), 1035$ (s), 913 (s), 834 (s), 774 (s), 671 (m); ${ }^{1} \mathrm{H}$ NMR (500 MHz, $\left.\mathrm{CDCl}_{3}\right) \delta 5.94(\mathrm{dd}, J=18.0,10.5 \mathrm{~Hz}, 1 \mathrm{H}), 4.97$ $(\mathrm{dd}, J=10.5,1.5 \mathrm{~Hz}, 1 \mathrm{H}), 4.97(\mathrm{dd}, J=18.0,1.5 \mathrm{~Hz}, 1 \mathrm{H}), 3.61(\mathrm{~d}, J=2.0 \mathrm{~Hz}, 1 \mathrm{H}), 2.67(\mathrm{~m}$, 1H), 1.77 (d, $J=2.5 \mathrm{~Hz}, 3 \mathrm{H}), 1.05$ (d, $J=7.0 \mathrm{~Hz}, 3 \mathrm{H}), 1.01(\mathrm{~s}, 3 \mathrm{H}), 1.00(\mathrm{~s}, 3 \mathrm{H}), 0.95$ (s, 9H), $0.18(\mathrm{~s}, 3 \mathrm{H}), 0.10(\mathrm{~s}, 3 \mathrm{H}) ;{ }^{13} \mathrm{C}\left(125.8 \mathrm{MHz}, \mathrm{CDCl}_{3}\right) \delta 146.0,111.6,85.8,82.4,75.8,43.3,28.0$, 26.5, 25.3, 23.8, 18.8, 16.2, 3.8, -3.0, -4.5; HRMS $m / z\left(E S I, M+\mathrm{Na}^{+}\right)$calcd 303.2115, found 303.2109 .

(+)-5-Acetoxy-dec-2-yne (6):

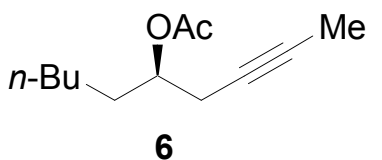

Propyne was bubbled through a solution of $n$-BuLi (2.5 M hexanes, $8 \mathrm{~mL}, 20 \mathrm{mmole})$ was added to cold $\left(-78^{\circ} \mathrm{C}\right) \mathrm{THF}(30 \mathrm{~mL})$ for 10 minutes, at which point $(R)$-1,2-heptene-oxide ${ }^{3,4}$ (1.26 g, $11.02 \mathrm{mmole}$ ) was added dropwise, followed by $\mathrm{Et}_{2} \mathrm{O} \cdot \mathrm{BF}_{3}(1.14 \mathrm{~mL}, 8.99 \mathrm{mmole})$. The reaction was stirred at $-78{ }^{\circ} \mathrm{C}$ for 1.5 hours, quenched with aqueous sodium bicarbonate and diethyl ether, the two layers were separated and the aqueous layer extracted with diethyl ether. The combined organics were dried over magnesium sulfate, concentrated and purified by silica gel chromatography (4:1 hexanes:diethyl ether) to give $1.52 \mathrm{~g}(94 \%)$ of the homopropargylic alcohol, a portion (694 mg, $4.5 \mathrm{mmole})$ of which was dissolved in DCM (9 mL) and cooled to 0 ${ }^{\circ} \mathrm{C}$. To this was added triethylamine $(1.88 \mathrm{~mL}, 13.5 \mathrm{mmole})$, acetic anhydride $(0.64 \mathrm{~mL}, 6.77$ mmole) and DMAP (55 $\mathrm{mg}, 0.45 \mathrm{mmole}$ ). The reaction was warmed to room temperature and stirred for 1.5 hours then quenched with saturated aqueous ammonium chloride. The aqueous phase was extracted with diethyl ether and the combined organics washed with (saturated aqueous) sodium bicarbonate, ammonium chloride, and sodium chloride. The organic phase was 
dried over magnesium sulfate, concentrated and purified by silica gel chromatography (15:1 hexanes:diethyl ether) to give $811 \mathrm{mg}(92 \%)$ of 6. $[\alpha]_{\mathrm{D}}+45.4\left(c 0.94,22{ }^{\circ} \mathrm{C}, \mathrm{CHCl}_{3}\right)$; IR 2957 (s), 2931 (s), 2861 (s), 1740 (s), 1436 (m), 1374 (s), 1239 (s), 1026(s); ${ }^{1} \mathrm{H}$ NMR (500 MHz, $\left.\mathrm{CDCl}_{3}\right) \delta 4.88(\mathrm{~m}, 1 \mathrm{H}), 2.39(\mathrm{~m}, 2 \mathrm{H}), 2.06(\mathrm{~s}, 3 \mathrm{H}), 1.78(\mathrm{t}, J=2.5 \mathrm{~Hz}, 3 \mathrm{H}), 1.65(\mathrm{~m}, 2 \mathrm{H}), 1.30$ (bm, 6H), $0.88(\mathrm{~m}, 3 \mathrm{H}) ;{ }^{13} \mathrm{C}\left(125.8 \mathrm{MHz}, \mathrm{CDCl}_{3}\right) \delta 170.9,77.8,74.6,72.6,33.2,31.8,25.1$, 24.4, 22.7, 21.4, 14.2, 3.7; HRMS $m / z\left(E S I, M+\mathrm{Na}^{+}\right)$calcd 219.1356, found 219.1357.

\section{2,2-Dimethyl-but-3-enal (16):}

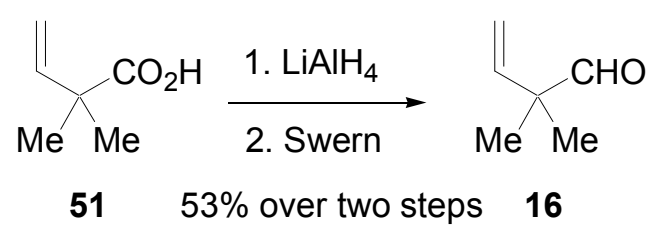

A solution of $\mathbf{5 1}^{5}(6.72 \mathrm{~g}, 58.9 \mathrm{mmole})$ in diethyl ether $(26 \mathrm{~mL})$ was added dropwise to a solution of $\mathrm{LiAlH}_{4}(2.05 \mathrm{~g}, 54.0 \mathrm{mmole})$ was dissolved in diethyl ether $(137 \mathrm{~mL})$, stirred for 2.5 hours then quenched with water. This resulted in the formation of an emulsion which was dissolved using $5 \mathrm{M} \mathrm{HCl}$. The aqueous layer was extracted with diethyl ether, the combined organics were washed with water and brine, dried over magnesium sulfate and concentrated ( 0 ${ }^{\circ} \mathrm{C}, 80$ torr). The residue was distilled at 0.1 torr, $35-45^{\circ} \mathrm{C}$ (receiver flask $=-78^{\circ} \mathrm{C}$ ) to give $4.4 \mathrm{~g}$ (75\%) of the primary alcohol, a portion of which was oxidized to 16 . Oxalyl chloride $(2.49 \mathrm{~mL}$, 30.0 mmole $)$ and DMSO $(2.84 \mathrm{~mL}, 40.0$ mmole $)$ were added to cold $\left(-78^{\circ} \mathrm{C}\right)$ DCM $(100 \mathrm{~mL})$ and the reaction stirred for 20 minutes. A solution of the primary alcohol (2.0 g, $20.0 \mathrm{mmole})$ in $15 \mathrm{~mL}$ DCM was added and the reaction stirred for an additional 30 minutes, followed by the addition of triethylamine $(8.4 \mathrm{~mL}, 60.0 \mathrm{mmole})$. The reaction was warmed to room temperature and stirred for 2 hours, then quenched via the addition of water. The layers were separated and the aqueous layer extracted with diethyl ether, the combined organics were washed with $0.5 \mathrm{M}$ $\mathrm{HCl}$, water, and brine then dried over magnesium sulfate. The solvent was removed under 
atmospheric pressure via distillation through a vigreux column $\left(75^{\circ} \mathrm{C}\right)$, THF $(4 \mathrm{~mL})$ was used to rinse the column back into the distillate flask and the majority of the THF was removed via distillation $\left(95^{\circ} \mathrm{C}\right)$. The receiver flask was then cooled to $-78{ }^{\circ} \mathrm{C}$ and $\mathbf{1 6}$ was brought over via vacuum transfer as a solution in THF. The composition of the solution was determined by NMR to be about $1.38 \mathrm{~g}$ of $\mathbf{1 6}(71 \%)$ in $1.3 \mathrm{~mL}$ THF (solution $\rho=0.94 \mathrm{~g} / \mathrm{ml}$ ). ${ }^{1} \mathrm{H}$ NMR (500 MHz, $\left.\mathrm{CDCl}_{3}\right) \delta 9.40(\mathrm{~s}, 1 \mathrm{H}), 5.81(\mathrm{dd}, J=17.5,10.5 \mathrm{~Hz}, 1 \mathrm{H}), 5.21(\mathrm{~d}, J=10.5 \mathrm{~Hz}, 1 \mathrm{H}), 5.15(\mathrm{~d}, J=$ $17.5 \mathrm{~Hz}, 1 \mathrm{H}), 1.20(\mathrm{~s}, 6 \mathrm{H})$.

\section{(-)-3,3,5-Trimethyl-hept-1-en-6-yn-4-ol (18):}

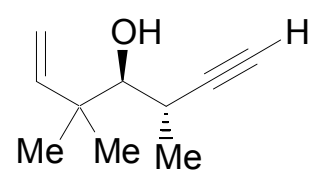

18

To a cold $\left(-78^{\circ} \mathrm{C}\right)$ orange solution of $\mathrm{Pd}(\mathrm{OAc})_{2}(5.6 \mathrm{mg}, 0.025 \mathrm{mmol})$ in $\mathrm{THF}(4 \mathrm{~mL})$ was added powdered $\mathrm{PPh}_{3}(6.6 \mathrm{mg}, 0.025 \mathrm{mmol})$ and the solution stirred until the $\mathrm{PPh}_{3}$ dissolved, at which point the solution turned yellow. $(R)-(+)-3$-Butyn-2-ol methansulfonate ${ }^{6}$ (96.3 g, $0.65 \mathrm{mmol})$ and $16(0.5 \mathrm{mmol})$ were added, followed by dropwise addition of diethylzinc ( $1 \mathrm{M}$ hexanes, $1.5 \mathrm{~mL}, 1.5 \mathrm{mmol}$ ) over 15 minutes. The reaction was stirred at -78 ${ }^{\circ} \mathrm{C}$ for 15 minutes and then placed in an ice bath, which was maintained at $0{ }^{\circ} \mathrm{C}$ for $2.5 \mathrm{~h}$, during which time the reaction solution turned dark. The reaction was quenched via the careful addition of saturated aqueous ammonium chloride, and then diluted with diethyl ether. The phases were separated and the aqueous layer extracted with diethyl ether. The combined organic layers were washed with brine and stirred for 20 minutes with magnesium sulfate and decolorizing agent. The slurry was filtered, concentrated and then purified by silica gel chromatography (12:1 hexanes/diethyl ether) to give $\mathbf{1 8}$ as a clear oil $\left(61.3 \mathrm{mg}, 80 \%, 90 \%\right.$ ee). $[\alpha]_{\mathrm{D}}-30.1(c 1.47,21$ ${ }^{\circ} \mathrm{C}, \mathrm{CHCl}_{3}$ ); chiral $\mathrm{GC}$ analysis (BDA column, $60{ }^{\circ} \mathrm{C}$ hold 5 min $\rightarrow 90{ }^{\circ} \mathrm{C}$ at $1{ }^{\circ} \mathrm{C} / \mathrm{min}, 0.9$ 
$\left.\mathrm{mL} / \mathrm{min} \mathrm{H}_{2}\right) \mathrm{R}_{\mathrm{F}}(R, R)=24.59 \min , \mathrm{R}_{\mathrm{F}}(S, S)=24.96 \mathrm{~min} ; \mathrm{IR} 3550(\mathrm{bm}), 3307$ (s), $2975(\mathrm{~s})$, $2876(\mathrm{~m}), 1638(\mathrm{~m}), 1452$ (m), $1383(\mathrm{~m}), 1118(\mathrm{~m}), 1051(\mathrm{~m}), 978(\mathrm{~s}), 917(\mathrm{~s}), 632$ (s); ${ }^{1} \mathrm{H}$ NMR $\left(500 \mathrm{MHz}, \mathrm{CDCl}_{3}\right) \delta 5.91(\mathrm{dd}, J=17.5,11.0 \mathrm{~Hz}, 1 \mathrm{H}), 5.08(\mathrm{dd}, J=11.0,1.0 \mathrm{~Hz}, 1 \mathrm{H}), 5.07$ (dd, $J=17.5,1.0 \mathrm{~Hz}, 1 \mathrm{H}), 3.10(\mathrm{dd}, J=9.5,1.2 \mathrm{~Hz}, 1 \mathrm{H}), 2.80\left(\mathrm{qd}, J_{\mathrm{q}}=7.0 \mathrm{~Hz}, J_{\mathrm{d}}=1.5 \mathrm{~Hz}, 1 \mathrm{H}\right), 2.20$ $(\mathrm{d}, J=2.5 \mathrm{~Hz}, 1 \mathrm{H}), 1.96(\mathrm{~d}, J=10.0 \mathrm{~Hz}, 1 \mathrm{H}), 1.30(\mathrm{~d}, J=7.0 \mathrm{~Hz}, 3 \mathrm{H}), 1.10(\mathrm{~s}, 3 \mathrm{H}), 1.09$ (s, $3 \mathrm{H}) ;{ }^{13} \mathrm{C}\left(125.8 \mathrm{MHz}, \mathrm{CDCl}_{3}\right) \delta 145.1,113.5,85.2,80.4,73.3,42.7,28.5,25.0,22.2,21.4$; HRMS $m / z$ (ESI, M+Na ${ }^{+}$) calcd 175.1093, found 175.1092.

\section{(-)-3,3,5-Trimethyl-4-(tert-butyldimethylsilyloxy)-hept-1-en-6-yn (52):}

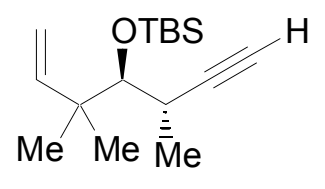

52

tert-Butyldimethylsilyltriflate (TBSOTf) $(1.09 \mathrm{~mL}, 5.16 \mathrm{mmole})$ was added to a cold ($\left.78{ }^{\circ} \mathrm{C}\right)$ solution of $18(240 \mathrm{mg}, 1.58 \mathrm{mmole})$ and 2,6-lutidine (1.1 mL, $\left.9.4 \mathrm{mmole}\right)$ in DCM (10 $\mathrm{mL}$ ), and the solution was warmed to room temperature overnight. Water and diethyl ether were added to the reaction, the aqueous layer was extracted with diethyl ether and the combined organic layers were washed with brine, dried over magnesium sulfate, concentrated, and purified by chromatography (hexanes) to give $374 \mathrm{mg}(89 \%)$ of $\mathbf{5 2}$ as a clear oil. $[\alpha]_{\mathrm{D}}-6.0(c 0.29,21$ ${ }^{\circ} \mathrm{C}, \mathrm{CHCl}_{3}$ ); IR 3314 (s), 3084 (w), 2960 (s), 2858 (s), 1640 (w), 1473 (s), 1415 (m), 1361 (s), 1253 (s), 1130 (s), 1091 (s), 1017 (s), 916 (s), 862 (s), 837 (s), 773 (s), 631 (s); ${ }^{1} \mathrm{H}$ NMR (500 $\left.\mathrm{MHz}, \mathrm{CDCl}_{3}\right) \delta 5.91(\mathrm{dd}, J=17.5,10.5 \mathrm{~Hz}, 1 \mathrm{H}), 5.02(\mathrm{dd}, J=17.5,1.5 \mathrm{~Hz}, 1 \mathrm{H}), 4.99(\mathrm{dd}, J=$ $10.5,1.5 \mathrm{~Hz}, 1 \mathrm{H}), 3.24(\mathrm{~d}, J=1.0 \mathrm{~Hz}, 1 \mathrm{H}), 2.76\left(\mathrm{ddq}, J_{\mathrm{q}}=7.5 \mathrm{~Hz}, J_{\mathrm{d}}=2.5,1.0 \mathrm{~Hz}, 1 \mathrm{H}\right), 2.03(\mathrm{~d}$, $J=2.5 \mathrm{~Hz}, 1 \mathrm{H}), 1.22(\mathrm{~d}, J=7.5 \mathrm{~Hz}, 3 \mathrm{H}), 1.09(\mathrm{~s}, 3 \mathrm{H}), 1.05(\mathrm{~s}, 3 \mathrm{H}), 0.96(\mathrm{~s}, 9 \mathrm{H}), 0.12(\mathrm{~s}, 3 \mathrm{H})$, $0.08(\mathrm{~s}, 3 \mathrm{H}) ;{ }^{13} \mathrm{C}\left(125.8 \mathrm{MHz}, \mathrm{CDCl}_{3}\right) \delta 146.4,112.0,86.9,82.0,71.0,43.5,29.4,26.4,25.8$, 22.4, 21.4, 18.9, -3.1, -3.5; HRMS $m / z\left(E S I, M+\mathrm{Na}^{+}\right)$calcd 289.1958, found 289.1958. 


\section{(-)-anti-1,6-Enyne (13):}

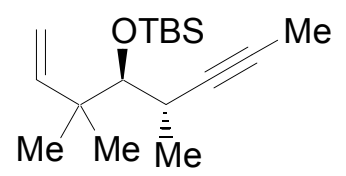

13

$n$-BuLi $(2.5 \mathrm{M}$ in hexanes, $2.6 \mathrm{~mL}, 6.5 \mathrm{mmol})$ was added dropwise to a cold $\left(-10{ }^{\circ} \mathrm{C}\right)$ solution of $i \operatorname{Pr}_{2} \mathrm{NH}(0.95 \mathrm{~mL}, 6.75 \mathrm{mmol})$ in $\mathrm{THF}(21 \mathrm{~mL})$ the solution was stirred for 15 minutes, then cooled to $-78^{\circ} \mathrm{C}$. $52(360 \mathrm{mg}, 1.35 \mathrm{mmol})$, DMPU (Aldrich, $1.39 \mathrm{~mL}, 11.5$ $\mathrm{mmol}$ ), and MeI (filtered through activated basic alumina, $0.40 \mathrm{~mL}, 6.5 \mathrm{mmol}$ ) were added to the solution at $-78^{\circ} \mathrm{C}$, the solution was warmed to $0{ }^{\circ} \mathrm{C}$ ( 5 minutes) then to room temperature $(2.5$ h). The reaction was quenched via the addition of water, and the product extracted with diethyl ether. The combined organic layers were washed with brine, dried over magnesium sulfate, filtered, concentrated and purified by silica gel chromatography (hexanes) to give $\mathbf{1 3}$ (360 mg, 95\%). $[\alpha]_{\mathrm{D}}-0.44\left(c 0.90,22{ }^{\circ} \mathrm{C}, \mathrm{CHCl}_{3}\right)$; IR 2959(s), 2859 (s), 1639 (w), 1473 (s), 1361 (s), 1255 (s), 1056 (s), 999 (s), 857 (s), 835 (s), 774 (s); ${ }^{1} \mathrm{H}$ NMR (500 MHz, $\left.\mathrm{CDCl}_{3}\right) \delta 5.92$ (dd, $J=$ $17.5,11.0 \mathrm{~Hz}, 1 \mathrm{H}), 4.99(\mathrm{dd}, J=17.4,1.5 \mathrm{~Hz}, 1 \mathrm{H}), 4.96(\mathrm{dd}, J=11.0,1.5 \mathrm{~Hz}, 1 \mathrm{H}), 3.21(\mathrm{~d}, J=$ $1.5 \mathrm{~Hz}, 1 \mathrm{H}), 2.67(\mathrm{~m}, 1 \mathrm{H}), 1.76(\mathrm{~d}, J=2.5 \mathrm{~Hz}, 3 \mathrm{H}), 1.16(\mathrm{~d}, J=7.5 \mathrm{~Hz}, 3 \mathrm{H}), 1.06(\mathrm{~s}, 3 \mathrm{H}), 1.03$ $(\mathrm{s}, 3 \mathrm{H}), 0.96(\mathrm{~s}, 9 \mathrm{H}), 0.11(\mathrm{~s}, 3 \mathrm{H}), 0.07(\mathrm{~s}, 3 \mathrm{H}) ;{ }^{13} \mathrm{C}\left(125.8 \mathrm{MHz}, \mathrm{CDCl}_{3}\right) \delta 146.7,111.5,82.3$, 81.7, 77.9, 43.4, 29.6, 26.3, 25.6, 22.7, 21.6, 18.8, 3.8, -3.3, -3.5; HRMS $m / z\left(E S I, M+\mathrm{Na}^{+}\right)$ calcd 303.2115, found 303.2115.

\section{$\delta$-Lactones $(53,21,54)$ :}



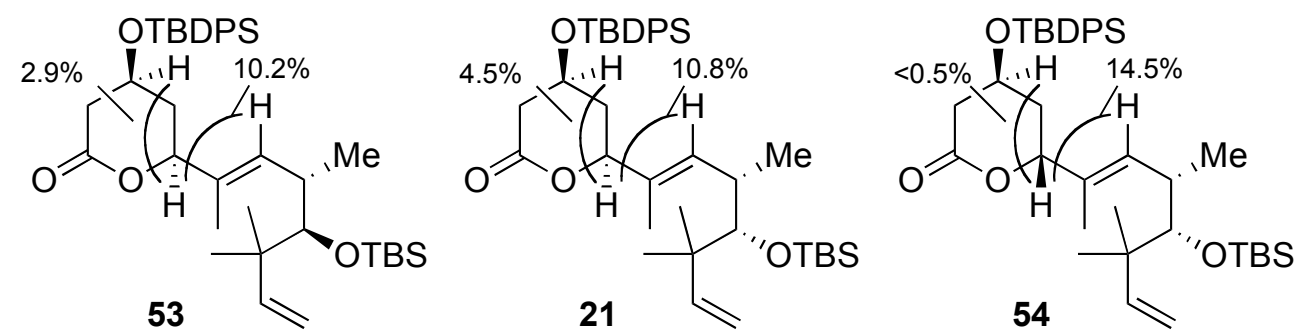

53:

$[\alpha]_{\mathrm{D}}+4.6\left(c 0.19,21^{\circ} \mathrm{C}, \mathrm{CHCl}_{3}\right)$; IR 2959 (s), 2931 (s), 2858 (s), $1744(\mathrm{~s}), 1472(\mathrm{~m})$, 1428 (m), 1380 (m), 1252 (m), 1106 (s), 1027 (s), 834 (m), 773 (m), 702 (s); ${ }^{1} \mathrm{H}$ NMR (500 $\left.\mathrm{MHz}, \mathrm{CDCl}_{3}\right) \delta 7.66(\mathrm{~m}, 4 \mathrm{H}), 7.47(\mathrm{~m}, 2 \mathrm{H}), 7.41(\mathrm{~m}, 4 \mathrm{H}), 5.90(\mathrm{dd}, J=17.5,10.9 \mathrm{~Hz}, 1 \mathrm{H}), 5.36$ (nOe 10.2\%) (d, $J=9.8 \mathrm{~Hz}, 1 \mathrm{H}), 4.98(\mathrm{dd}, J=17.5,1.4 \mathrm{~Hz}, 1 \mathrm{H}), 4.96(\mathrm{dd}, J=10.9,1.4 \mathrm{~Hz}, 1 \mathrm{H})$, $4.27($ nOe $10.2 \%, 2.9 \%)(\mathrm{dd}, J=11.9,3.0 \mathrm{~Hz}, 1 \mathrm{H}), 4.12(\mathrm{nOe} 2.9 \%)(\mathrm{m}, 1 \mathrm{H}), 3.28(\mathrm{~d}, J=1.9$ Hz, 1H), 2.71 (ddd, $J=17.2,5.8,1.3 \mathrm{~Hz}, 1 \mathrm{H}), 2.62(\mathrm{~m}, 1 \mathrm{H}), 2.49$ (dd, $J=17.3,8.3 \mathrm{~Hz}, 1 \mathrm{H})$, $1.93(\mathrm{~m}, 1 \mathrm{H}), 1.84(\mathrm{~m}, 1 \mathrm{H}), 1.58(\mathrm{~d}, J=1.0 \mathrm{~Hz}, 3 \mathrm{H}), 1.07$ (s, 9H), $1.00(\mathrm{~s}, 3 \mathrm{H}), 0.98(\mathrm{~s}, 3 \mathrm{H}), 0.94$ (s, 9H), $0.86(\mathrm{~d}, J=6.5 \mathrm{~Hz}, 3 \mathrm{H}), 0.04(\mathrm{~s}, 3 \mathrm{H}), 0.03(\mathrm{~s}, 3 \mathrm{H}) ;{ }^{13} \mathrm{C}\left(125.8 \mathrm{MHz}, \mathrm{CDCl}_{3}\right) \delta 171.0$, $146.5,136.3,135.9,133.6,133.3,130.2,130.2,129.3,128.1,128.0,111.4,82.5,82.2,65.7,43.5$, $40.1,37.4,34.2,27.0,26.6,25.6,23.7,19.2,18.9,15.7,11.7,-2.5,-3.7$; HRMS $m / z$ (ESI, $\mathrm{M}+\mathrm{H}^{+}$) calcd 635.3946, found 635.3962.

21:

$[\alpha]_{\mathrm{D}}+22.4\left(c 0.87,21{ }^{\circ} \mathrm{C}, \mathrm{CHCl}_{3}\right) ; \mathrm{IR} 2959(\mathrm{~s}), 2931$ (s), $2858(\mathrm{~s}), 1742(\mathrm{~s}), 1472(\mathrm{~m})$, 1428 (s), 1380 (m), 1253 (s), 1107 (s), 1036 (s), 911 (s), 834 (s), 736 (s), 702 (s); ${ }^{1} \mathrm{H}$ NMR (500 $\left.\mathrm{MHz}, \mathrm{CDCl}_{3}\right) \delta 7.66(\mathrm{~m}, 4 \mathrm{H}), 7.46(\mathrm{~m}, 2 \mathrm{H}), 7.40(\mathrm{~m}, 4 \mathrm{H}), 5.79(\mathrm{dd}, J=17.5,10.9 \mathrm{~Hz}, 1 \mathrm{H}), 5.65$ (nOe 10.8\%) (d, $J=9.4 \mathrm{~Hz}, 1 \mathrm{H}), 4.92(\mathrm{dd}, J=17.5,1.4 \mathrm{~Hz}, 1 \mathrm{H}), 4.89$ (dd, $J=10.9,1.4 \mathrm{~Hz}, 1 \mathrm{H})$, $4.27($ nOe $10.2 \%, 4.5 \%)(\mathrm{dd}, J=11.9,3.0 \mathrm{~Hz}, 1 \mathrm{H}), 4.12(\mathrm{nOe} 4.5 \%)(\mathrm{m}, 1 \mathrm{H}), 3.35$ (d, $J=0.6$ $\mathrm{Hz}, 1 \mathrm{H}$ ), 2.72 (ddd, $J=17.3,5.9,1.3 \mathrm{~Hz}, 1 \mathrm{H}$ ), 2.67 (quint, $J=7.5 \mathrm{~Hz}, 1 \mathrm{H}$ ), 2.49 (dd, $J=17.3$, $8.3 \mathrm{~Hz}, 1 \mathrm{H}), 1.91(\mathrm{~m}, 1 \mathrm{H}), 1.84(\mathrm{~m}, 1 \mathrm{H}), 1.58(\mathrm{~d}, J=1.0 \mathrm{~Hz}, 3 \mathrm{H}), 1.07(\mathrm{~s}, 9 \mathrm{H}), 0.95(\mathrm{~s}, 9 \mathrm{H}), 0.94$ 
(s, 3H), $0.92(\mathrm{~d}, J=7.5 \mathrm{~Hz}, 3 \mathrm{H}), 0.90(\mathrm{~s}, 3 \mathrm{H}), 0.10(\mathrm{~s}, 3 \mathrm{H}), 0.09(\mathrm{~s}, 3 \mathrm{H}) ;{ }^{13} \mathrm{C}(125.8 \mathrm{MHz}$, $\left.\mathrm{CDCl}_{3}\right) \delta 171.1,146.7,135.9,133.6,133.4,132.2,130.3,130.2,129.2,128.1,128.0,111.4,84.1$ $82.4,65.7,43.2,40.1,37.3,34.5,27.0,26.6,26.2,22.5,21.2,19.2,19.0,12.2,-2.8,-3.2$; HRMS $m / z\left(\mathrm{ESI}, \mathrm{M}+\mathrm{H}^{+}\right)$calcd 635.3946, found 635.3961.

54:

$[\alpha]_{\mathrm{D}}+5.9\left(c 0.38,22{ }^{\circ} \mathrm{C}, \mathrm{CHCl}_{3}\right)$; IR 2959 (s), 2930 (s), 2858 (s), 1739 (s), $1472(\mathrm{~m})$, $1428(\mathrm{~m}), 1235$ (s), 1111 (s), 1037 (s), 870 (m), 773 (m), 702 (s); ${ }^{1} \mathrm{H}$ NMR (500 MHz, $\left.\mathrm{CDCl}_{3}\right) \delta$ $7.64(\mathrm{~m}, 4 \mathrm{H}), 7.46(\mathrm{~m}, 2 \mathrm{H}), 7.40(\mathrm{~m}, 4 \mathrm{H}), 5.84(\mathrm{dd}, J=17.6,10.7 \mathrm{~Hz}, 1 \mathrm{H}), 5.79(\mathrm{nOe} 14.5 \%)(\mathrm{d}$, $J=9.4 \mathrm{~Hz}, 1 \mathrm{H}), 5.24(\mathrm{nOe} 14.5 \%)(\mathrm{dd}, J=9.9,5.5 \mathrm{~Hz}, 1 \mathrm{H}), 4.96(\mathrm{dd}, J=17.6,1.1 \mathrm{~Hz}, 1 \mathrm{H})$, $4.84(\mathrm{dd}, J=10.7,1.1 \mathrm{~Hz}, 1 \mathrm{H}), 4.28(\mathrm{~m}, 1 \mathrm{H}), 3.37(\mathrm{~s}, 1 \mathrm{H}), 2.65(\mathrm{~m}, 1 \mathrm{H}), 2.45(\mathrm{dd}, J=17.6,4.3$ $\mathrm{Hz}, 1 \mathrm{H}), 1.68(\mathrm{~m}, 2 \mathrm{H}), 1.53$ (s, 3H), $1.08(\mathrm{~s}, 9 \mathrm{H}), 0.99$ (d, $J=7.0 \mathrm{~Hz}, 3 \mathrm{H}), 0.96$ (s, 9H), 0.96 (m, $3 \mathrm{H}), 0.91(\mathrm{~s}, 3 \mathrm{H}), 0.12(\mathrm{~s}, 3 \mathrm{H}), 0.11(\mathrm{~s}, 3 \mathrm{H}) ;{ }^{13} \mathrm{C}\left(125.8 \mathrm{MHz}, \mathrm{CDCl}_{3}\right) \delta$ 170.7, 146.7, 135.9, 135.8, 133.4, 132.6, 130.3, 130.2, 129.3, 128.5, 128.1, 128.1, 111.0, 84.3, 82.6, 64.7, 43.2, 39.0, $34.5,34.0,27.1,26.7,26.0,23.2,21.2,19.3,19.1,11.4,-2.8,-3.2$; HRMS $m / z\left(E S I, M+\mathrm{H}^{+}\right)$ calcd 635.3946, found 635.3967.

(+)-Enyne (22):

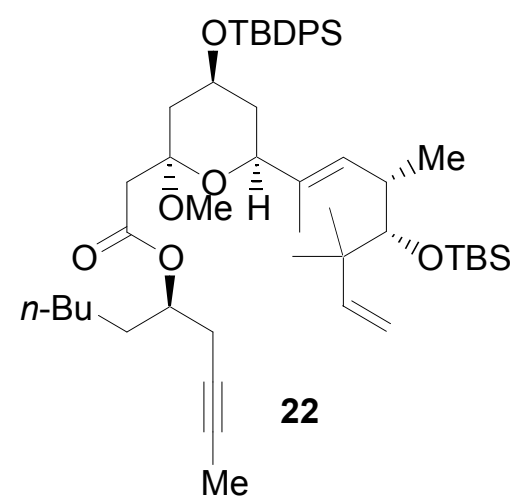


$n$-BuLi $(2.5 \mathrm{M}$ in hexanes, $492 \mu \mathrm{L}, 1.23 \mathrm{mmol})$ was added to a cold $\left(-10^{\circ} \mathrm{C}\right)$ solution of $i \operatorname{Pr}_{2} \mathrm{NH}(173 \mu \mathrm{L}, 1.22 \mathrm{mmol})$ in THF $(3 \mathrm{~mL})$ the solution was stirred for 15 minutes, then cooled to $-42{ }^{\circ} \mathrm{C}$. A solution of $6(236 \mathrm{mg}, 1.2 \mathrm{mmole})$ in THF $(1 \mathrm{~mL})$ was added dropwise and the solution stirred for 45 minutes, followed by the dropwise addition of $\mathbf{2 0}$ (c. 62:38 mixture of diastereomers, $220 \mathrm{mg}, 0.33 \mathrm{mmole})$ in THF $(2 \mathrm{~mL})$. After 4 hours at $-42{ }^{\circ} \mathrm{C}$ the reaction was quenched via the addition of water and diethyl ether. The layers were separated and the aqueous layer extracted with diethyl ether. The combined organic layers were washed with $0.5 \mathrm{M} \mathrm{HCl}$, water, and brine then dried over magnesium sulfate and concentrated. The crude material was flushed through a plug of silica eluting with 10:1 hexanes:diethyl ether leaving a clear oil. This crude material was dissolved in $7 \mathrm{~mL}$ of methanol and heated to $65^{\circ} \mathrm{C}$ for 2 hours with citric acid (100 mg, $0.48 \mathrm{mmole}$ ), the methanol was removed in vacuo and the residue purified by silica gel chromatography (30:1 hexanes:ethyl acetate) to give a clear oil. The oil was warmed to $50{ }^{\circ} \mathrm{C}$ and placed under vacuum (0.04 torr) overnight to remove excess 6, resulting in $160 \mathrm{mg}(57 \%)$ of $22(>95: 5 \mathrm{dr}) .[\alpha]_{\mathrm{D}}+32.8\left(c 0.57,22{ }^{\circ} \mathrm{C}, \mathrm{CHCl}_{3}\right)$; IR $3584(\mathrm{~m}), 2956(\mathrm{~s}), 2930(\mathrm{~s}), 2858(\mathrm{~s})$, 1737 (s), 1472 (m), 1428 (m), 1378 (m), 1251 (m), 1112 (s), 1042 (s), 834 (m), 702 (s); ${ }^{1} \mathrm{H}$ NMR $\left(500 \mathrm{MHz}, \mathrm{CDCl}_{3}\right) \delta 7.66(\mathrm{~m}, 4 \mathrm{H}), 7.41(\mathrm{~m}, 2 \mathrm{H}), 7.37(\mathrm{~m}, 4 \mathrm{H}), 5.81(\mathrm{dd}, J=17.5,10.5 \mathrm{~Hz}, 1 \mathrm{H})$, $5.53(\mathrm{~d}, J=9.5 \mathrm{~Hz}, 1 \mathrm{H}), 4.87(\mathrm{~m}, 3 \mathrm{H}), 4.14(\mathrm{~m}, 1 \mathrm{H}) 3.58(\mathrm{~d}, J=11.0 \mathrm{~Hz}, 1 \mathrm{H}), 3.33(\mathrm{~s}, 1 \mathrm{H}), 2.73$ (d, $J=13.5 \mathrm{~Hz}, 1 \mathrm{H}), 2.61(\mathrm{~m}, 1 \mathrm{H}), 2.54(\mathrm{~d}, J=13.5 \mathrm{~Hz}, 1 \mathrm{H}), 2.37(\mathrm{~m}, 2 \mathrm{H}), 2.26(\mathrm{dd}, J=13.0$ $4.0 \mathrm{~Hz}, 1 \mathrm{H}), 1.77$ (t, $J=2.5 \mathrm{~Hz}, 3 \mathrm{H}), 1.69$ (m, 2H), 1.49 (s, 3H), 1.42 (q, $J=12.0 \mathrm{~Hz}, 1 \mathrm{H}), 1.36-$ $1.24(\mathrm{~m}, 9 \mathrm{H}), 1.05(\mathrm{~s}, 9 \mathrm{H}), 0.94(\mathrm{~s}, 9 \mathrm{H}), 0.94(\mathrm{~m}, 3 \mathrm{H}), 0.89(\mathrm{~m}, 11 \mathrm{H}), 0.09(\mathrm{~s}, 3 \mathrm{H}), 0.08(\mathrm{~s}, 3 \mathrm{H})$ ${ }^{13} \mathrm{C}\left(125.8 \mathrm{MHz}, \mathrm{CDCl}_{3}\right) \delta 169.1,147.0,136.0,136.0,134.8,134.6,131.5,129.7,129.4,127.7$, 127.7, 111.0, 99.6, 84.3, 77.8, 74.7, 74.2, 72.7, 67.0, 47.8, 43.2, 43.0, 42.6, 38.8, 34.4, 33.1, 31.8, 
27.2, 26.6, 26.1, 25.0, 24.2, 22.8, 22.7, 21.3, 19.3, 19.0, 14.2, 12.9, 3.8, -2.8, -3.2; HRMS m/z (ESI, $\mathrm{M}+\mathrm{Na}^{+}$) calcd 867.5386, found 867.5473.

\section{(-)-5-Hydroxy-4,4,6-trimethyl-oct-7-yn-3-one (27):}

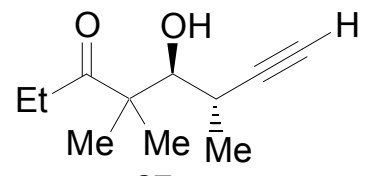

27

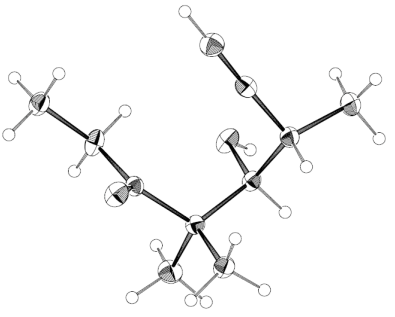

To a cold $\left(-78^{\circ} \mathrm{C}\right)$ orange solution of $\mathrm{Pd}(\mathrm{OAc})_{2}(65 \mathrm{mg}, 0.29 \mathrm{mmol})$ in THF $(120 \mathrm{~mL})$ was added powdered $\mathrm{PPh}_{3}(76 \mathrm{mg}, 0.29 \mathrm{mmol})$ and the solution stirred until it dissolved, at which point the solution turned yellow. $(R)-(+)-3$-Butyn-2-ol methansulfonate $(3.0 \mathrm{~g}, 20.2$ mmol) and 2,2-dimethyl-3-pentanon-al ${ }^{7}$ (1.99 g, $\left.15.5 \mathrm{mmol}\right)$ were added, followed by dropwise addition of diethylzinc ( $1 \mathrm{M}$ hexanes, $58 \mathrm{~mL}, 58 \mathrm{mmol})$ over 15 minutes. The reaction was stirred at $-78^{\circ} \mathrm{C}$ for 15 minutes and then placed in an ice bath, which was maintained at $0{ }^{\circ} \mathrm{C}$ for $2.5 \mathrm{~h}$, during which time the reaction solution turned dark. The reaction was quenched via the careful addition of saturated aqueous ammonium chloride, and then diluted with diethyl ether. The phases were separated and the aqueous layer extracted with diethyl ether. The combined organic layers were washed with brine and stirred for 20 minutes with magnesium sulfate and decolorizing agent. The slurry was filtered, concentrated and then purified by silica gel chromatography (3:1 hexanes/diethyl ether) to give a clear oil which crystallized upon standing (2.69 g, 95\%, > 95:5 dr, 90\% ee). Relative stereochemistry of 27 was determined by X-ray crystallography (see CIF file in supporting information). $[\alpha]_{\mathrm{D}}-11.1\left(c 0.81,21{ }^{\circ} \mathrm{C}, \mathrm{CHCl}_{3}\right)$; chiral GC analysis $\left(\beta-\mathrm{PH}\right.$ column, $\left.88^{\circ} \mathrm{C}, 1.5 \mathrm{ml} / \mathrm{min}_{2}\right) \mathrm{R}_{\mathrm{T}}(S, S)=46.85 \mathrm{~min}, \mathrm{R}_{\mathrm{T}}(R, R)=47.91$ 
min; IR 3415 (bs), 3265 (s), 2975 (s), 1681 (s), 1386 (m), 972 (m); ${ }^{1} \mathrm{H}$ NMR (500 MHz, $\mathrm{CDCl}_{3}$ ) $\delta 3.50(\mathrm{dd}, J=9.0,2.0 \mathrm{~Hz}, 1 \mathrm{H}), 3.35(\mathrm{~d}, J=9.0 \mathrm{~Hz}, 1 \mathrm{H}), 2.70(\mathrm{~m}, 1 \mathrm{H}), 2.65\left(\mathrm{dq}, J_{\mathrm{d}}=18.5, J_{\mathrm{q}}=\right.$ $7.0 \mathrm{~Hz}, 1 \mathrm{H}), 2.54\left(\mathrm{dq}, J_{\mathrm{d}}=18.5, J_{\mathrm{q}}=7.0 \mathrm{~Hz}, 1 \mathrm{H}\right), 2.14(\mathrm{~d}, J=2.0 \mathrm{~Hz}, 1 \mathrm{H}), 1.32(\mathrm{~d}, J=7.0 \mathrm{~Hz}$, 3H), 1.27 (s, 3H), $1.22(\mathrm{~s}, 3 \mathrm{H}), 1.02(\mathrm{t}, J=7.0 \mathrm{~Hz}, 3 \mathrm{H}) ;{ }^{13} \mathrm{C}\left(125.8 \mathrm{MHz}, \mathrm{CDCl}_{3}\right) \delta 218.1,84.9$, 73.0, 51.1, 32.4, 28.9, 23.5, 21.5, 20.4, 7.8; HRMS $m / z\left(E S I, M+\mathrm{Na}^{+}\right)$calcd 205.1205, found 205.1195 .

\section{(+)-3,5,5-Trimethyl-4,6-bis-triethylsilanyloxy-oct-5-en-1-yne (55):}

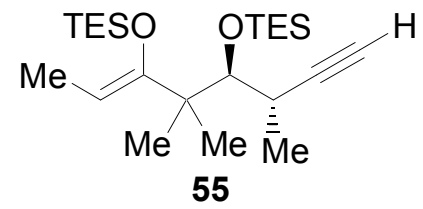

To a cold $\left(-78{ }^{\circ} \mathrm{C}\right)$ solution of $27(1.129 \mathrm{~g}, 6.2 \mathrm{mmol})$ and $\mathrm{NEt}_{3}(4.3 \mathrm{~mL}, 30.8 \mathrm{mmol})$ in DCM (12 mL) was added triethylsilyltriflate (TESOTf) $(5.6 \mathrm{~mL}, 24.8 \mathrm{mmol})$, the reaction was warmed to room temperature and stirred overnight. The reaction was quenched with saturated sodium bicarbonate and diluted with diethyl ether, the layers were separated and the organic layer was washed with brine, dried over magnesium sulfate, filtered, concentrated and purified by silica gel chromatography (hexanes) to give 55 as a clear oil $(2.49 \mathrm{~g}, 98 \%) .[\alpha]_{\mathrm{D}}+9.4(c 4.08$, $21^{\circ} \mathrm{C}, \mathrm{CHCl}_{3}$ ); IR 3314 (m), 2956 (s), 2878 (s), 1664 (m), 1459 (m), 1317 (m), 1240 (m), 1136 (s), 1008 (s), $738(\mathrm{~s}) ;{ }^{1} \mathrm{H}$ NMR (500 MHz, $\left.\mathrm{CDCl}_{3}\right) \delta 4.65$ (q, $\left.J=7.0 \mathrm{~Hz}, 1 \mathrm{H}\right), 3.66(\mathrm{~d}, J=1.0$ $\mathrm{Hz}, 1 \mathrm{H}), 2.72\left(\mathrm{ddq}, J_{\mathrm{q}}=7.0, J_{\mathrm{d}}=2.5,1.5 \mathrm{~Hz}, 1 \mathrm{H}\right), 2.00(\mathrm{~d}, J=2.5 \mathrm{~Hz}, 1 \mathrm{H}), 1.52(\mathrm{~d}, J=7.0 \mathrm{~Hz}$, 3H), $1.22(\mathrm{~d}, J=7.0 \mathrm{~Hz}, 3 \mathrm{H}), 1.09(\mathrm{~s}, 3 \mathrm{H}), 1.06(\mathrm{~s}, 3 \mathrm{H}), 1.01(\mathrm{t}, J=8.0 \mathrm{~Hz}, 9 \mathrm{H}), 1.00(\mathrm{t}, J=8.0$ $\mathrm{Hz}, 9 \mathrm{H}), 0.73(\mathrm{q}, J=8.0 \mathrm{~Hz}, 6 \mathrm{H}), 0.66$ (apparent dt, $\left.J_{\mathrm{t}}=8.0, J_{\mathrm{d}}=1.5 \mathrm{~Hz}, 6 \mathrm{H}\right) ;{ }^{13} \mathrm{C}(125.8 \mathrm{MHz}$, 
$\left.\mathrm{CDCl}_{3}\right) \delta 157.2,99.5,87.5,79.4,70.4,46.5,28.9,25.4,21.9,20.3,11.7,7.4,7.2,6.4,5.9$; HRMS $m / z$ (ESI, $\mathrm{M}+\mathrm{Na}^{+}$) calcd 433.2929, found 433.2911.

\section{(+)-4,6,6-Trimethyl-5,7-bis-triethylsilanyloxy-non-7-en-2-yne (25):}

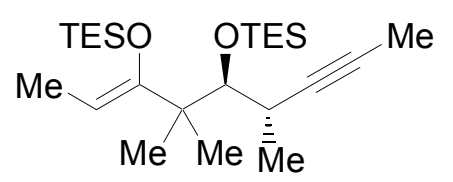

25

$n$-BuLi $(2.5 \mathrm{M}$ in hexanes, $10.8 \mathrm{~mL}, 27 \mathrm{mmol})$ was added dropwise to a cold $\left(-10{ }^{\circ} \mathrm{C}\right)$ solution of $i \operatorname{Pr}_{2} \mathrm{NH}(3.96 \mathrm{~mL}, 28.2 \mathrm{mmol})$ in $\mathrm{THF}(100 \mathrm{~mL})$ the solution was stirred for 15 minutes, then cooled to $-78^{\circ} \mathrm{C} .55$ (2.42 g, $\left.5.88 \mathrm{mmol}\right)$, DMPU (Aldrich, $5.92 \mathrm{~mL}, 48.8 \mathrm{mmol}$ ), and MeI (filtered through activated basic alumina, $1.75 \mathrm{~mL}, 28.1 \mathrm{mmol}$ ) were added to the solution at $-78{ }^{\circ} \mathrm{C}$, the solution was warmed to $0{ }^{\circ} \mathrm{C}$ (5 minutes) then to room temperature $(2.5$ h). The reaction was quenched via the addition of water, and the product extracted with diethyl ether. The combined organic layers were washed with brine, dried over magnesium sulfate, filtered, concentrated and purified by chromatography (hexanes) to give $25(2.26 \mathrm{~g}, 91 \%)$. [ $\alpha]_{\mathrm{D}}$ +11.0 (c 3.1, $21^{\circ} \mathrm{C}, \mathrm{CHCl}_{3}$ ); IR 2956 (s), 2916 (s), 2878 (s), 2361 (w), 1664 (m), 1458 (m), 1317 (m), 1116 (s), 1004 (s), 845 (m), 737 (s); ${ }^{1} \mathrm{H}$ NMR (500 MHz, $\left.\mathrm{CDCl}_{3}\right) \delta 4.62$ (q, $\left.J=6.5 \mathrm{~Hz}, 1 \mathrm{H}\right)$, $3.63(\mathrm{~d}, J=1.5 \mathrm{~Hz}, 1 \mathrm{H}), 2.63(\mathrm{~m}, 1 \mathrm{H}), 1.76(\mathrm{~d}, J=2.0 \mathrm{~Hz}, 1 \mathrm{H}), 1.50(\mathrm{~d}, J=7.0 \mathrm{~Hz}, 3 \mathrm{H}), 1.16$ $(\mathrm{d}, J=7.5 \mathrm{~Hz}, 3 \mathrm{H}), 1.06(\mathrm{~s}, 3 \mathrm{H}), 1.05(\mathrm{~s}, 3 \mathrm{H}), 1.00(\mathrm{t}, J=8.0 \mathrm{~Hz}, 9 \mathrm{H}), 1.00(\mathrm{t}, J=8.0 \mathrm{~Hz}, 9 \mathrm{H})$, $0.72(\mathrm{q}, J=8.0 \mathrm{~Hz}, 6 \mathrm{H}), 0.64(\mathrm{q}, J=8.0,6 \mathrm{H}) ;{ }^{13} \mathrm{C}\left(125.8 \mathrm{MHz}, \mathrm{CDCl}_{3}\right) \delta 157.4,99.3,82.2$,

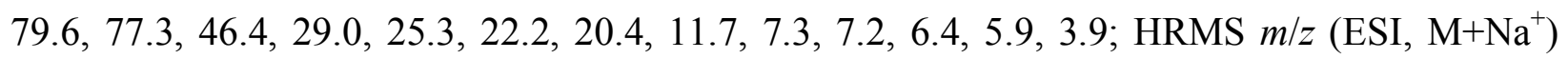
calcd 447.3085, found 447.3076.

(+)-ס-Lactone-silyl-enol-ether (29): 


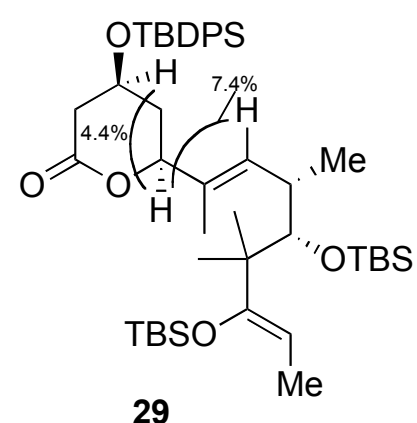

29

29: (77\% yield, $73: 27 \mathrm{dr})[\alpha]_{\mathrm{D}}+19.2\left(\mathrm{c} 2.72,21^{\circ} \mathrm{C}, \mathrm{CHCl}_{3}\right)$; IR 2958 (s), 2859 (s), 2252 (w), 1740 (w), 1472 (m), 1256 (s), 1107 (s), 909 (s); ${ }^{1} \mathrm{H}$ NMR (500 MHz, $\left.\mathrm{CDCl}_{3}\right) \delta 7.66$ (m, 4H), $7.46(\mathrm{~m}, 2 \mathrm{H}), 7.40(\mathrm{~m}, 4 \mathrm{H}), 5.67($ nOe $7.4 \%)(\mathrm{d}, J=9.5 \mathrm{~Hz}, 1 \mathrm{H}), 4.56(\mathrm{q}, J=7.0 \mathrm{~Hz}, 1 \mathrm{H})$, $4.28(\mathrm{nOe} 7.4 \%, 4.4 \%)(\mathrm{dd}, J=6.5,3.5 \mathrm{~Hz}, 1 \mathrm{H}), 4.12(\mathrm{nOe} 4.4 \%)(\mathrm{m}, 1 \mathrm{H}), 3.83(\mathrm{~s}, 1 \mathrm{H}), 2.71$ $(\mathrm{dd}, J=17.5,6.0 \mathrm{~Hz}, 1 \mathrm{H}), 2.66(\mathrm{~m}, 1 \mathrm{H}), 2.49(\mathrm{dd}, J=17.5,8.0 \mathrm{~Hz}, 1 \mathrm{H}), 1.86(\mathrm{~m}, 2 \mathrm{H}), 1.56(\mathrm{~d}, J$ $=1.0 \mathrm{~Hz}, 3 \mathrm{H}), 1.52(\mathrm{~d}, J=6.5 \mathrm{~Hz}, 3 \mathrm{H}), 1.07(\mathrm{~s}, 9 \mathrm{H}), 1.00(\mathrm{~s}, 9 \mathrm{H}), 0.99(\mathrm{~s}, 3 \mathrm{H}), 0.93(\mathrm{~s}, 9 \mathrm{H})$, $0.91(\mathrm{~d}, J=7.5 \mathrm{~Hz}, 3 \mathrm{H}), 0.84(\mathrm{~s}, 3 \mathrm{H}), 0.21(\mathrm{~s}, 3 \mathrm{H}), 0.20(\mathrm{~s}, 3 \mathrm{H}), 0.09(\mathrm{~s}, 3 \mathrm{H}), 0.07(\mathrm{~s}, 3 \mathrm{H}) ;{ }^{13} \mathrm{C}$ $\left(125.8 \mathrm{MHz}, \mathrm{CDCl}_{3}\right) \delta 171.2,157.3,135.9,133.6,133.4,132.8,130.3,130.2,128.9,128.1$, $128.0,99.2,82.8,65.7,46.4,40.1,37.3,34.2,27.0,26.8,26.8,26.6,26.4,21.4,21.2,19.4,19.2$, 19.0, 12.0, 11.8, -2.4, $-2.5,-2.9,-3.3 ;$ HRMS $m / z$ (ESI, $\left.\mathrm{M}+\mathrm{Na}^{+}\right)$calcd 801.4742, found 801.4735.

\section{(+)-Non-1-en-4-ol (56):}

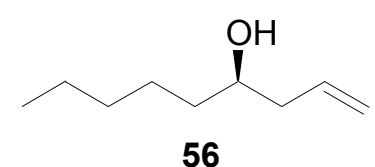

Copper (I) iodide (78 $\mathrm{mg}, 0.4 \mathrm{mmol}$ ) was placed in a round bottomed flask and cooled to $-78^{\circ} \mathrm{C}$. To this was added vinyl magnesium bromide generated from magnesium (535 mg, 22 
mmol) and an excess of vinyl bromide (1 M, THF). The vessel was warmed to $-10{ }^{\circ} \mathrm{C}$ and $(R)$ 1,2-heptene-oxide $(1.14 \mathrm{~g}, 10 \mathrm{mmol})$ in THF $(3.0 \mathrm{~mL})$ was added dropwise. The reaction was allowed to gradually warm to $0{ }^{\circ} \mathrm{C}$ over 1.5 hours and then carefully quenched via the addition of saturated aqueous ammonium chloride. The solution was extracted with diethyl ether and the combined organics were dried over magnesium sulfate, filtered, concentrated $\left(0{ }^{\circ} \mathrm{C}, 40 \mathrm{mmHg}\right)$, and purified by silica gel chromatography (10:1 hexanes/diethyl ether) to give $976 \mathrm{mg}(69 \%)$ of 56 as a clear oil. Spectral data matched that known in the literature. ${ }^{3}[\alpha]_{\mathrm{D}}+7.4\left(c 0.9,21{ }^{\circ} \mathrm{C}\right.$, $\left.\mathrm{CHCl}_{3}\right) ;{ }^{1} \mathrm{H}$ NMR $\left(500 \mathrm{MHz}, \mathrm{CDCl}_{3}\right) \delta 5.84(\mathrm{~m}, 1 \mathrm{H}), 5.15(\mathrm{~m}, 2 \mathrm{H}), 3.66(\mathrm{~m}, 1 \mathrm{H}), 2.32(\mathrm{~m}, 1 \mathrm{H})$, $2.14(\mathrm{~m}, 1 \mathrm{H}), 1.40-1.26(\mathrm{bm}, 8 \mathrm{H}), 0.90(\mathrm{t}, J=7.0 \mathrm{~Hz}, 3 \mathrm{H}) ;{ }^{13} \mathrm{C}\left(125.8 \mathrm{MHz}, \mathrm{CDCl}_{3}\right) \delta 135.1$, $118.3,70.9,42.2,37.0,32.1,25.6,22.8,14.3$.

\section{(-)-1-Triethylsiloxy-3-acetoxy-octane (30):}

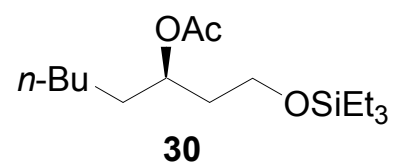

To a cold $\left(0^{\circ} \mathrm{C}\right)$ solution of $56(142 \mathrm{mg}, 1.0 \mathrm{mmole})$ in DCM (3 mL) was added triethylamine $(418 \mu \mathrm{L}, 3.0$ mmole $)$, acetic anhydride (142 $\mu \mathrm{L}, 1.5 \mathrm{mmole})$ and DMAP (12.2 mg, $0.1 \mathrm{mmole})$, this was warmed to room temperature and stirred for 1 hour. Saturated aqueous ammonium chloride and diethyl ether were added and the phases separated, the aqueous phase was extracted with diethyl ether and the combined organics washed with brine and dried over magnesium sulfate. The residual solvent was removed $\left(0{ }^{\circ} \mathrm{C}, 70\right.$ torr $)$ and the residue purified by silica gel chromatography (4:1 hexanes:diethyl ether) to give $183 \mathrm{mg}(100 \%)$ of the acetate protected alcohol a portion of which was used in the subsequent steps. Ozone was bubbled through a solution of the acetate protected alcohol $(60.4 \mathrm{mg}, 0.33 \mathrm{mmole})$ in methanol $(2 \mathrm{~mL})$ at 
$-78{ }^{\circ} \mathrm{C}$ until the solution turned blue, at which point the ozone was removed by bubbling argon through the solution until it was colorless. $\mathrm{NaBH}_{4}(25 \mathrm{mg}$ portions) was added until TLC determined that the reactant had been fully reduced to the primary alcohol. Saturated aqueous ammonium chloride was added and the product extracted with ethyl acetate. The combined organic layers were washed with brine, dried over magnesium sulfate and concentrated. The residue was dissolved in DMF $(1 \mathrm{~mL})$ and cooled to $0{ }^{\circ} \mathrm{C}$, to this was added chlorotriethylsilane ( $83 \mu \mathrm{L}, 0.49 \mathrm{mmole}$ ) and imidazole ( $40 \mathrm{mg}, 0.59 \mathrm{mmole})$ and the reaction was warmed to room temperature and stirred for 2 hours. Water and ethyl acetate were added and the aqueous phase extracted with ethyl acetate, the combined organics were washed with brine, dried over magnesium sulfate, concentrated and purified by silica gel chromatography (10:1 hexanes:diethyl ether) to give $66 \mathrm{mg}\left(68 \%\right.$ over two steps) of $\mathbf{3 0}$ as a clear oil. $[\alpha]_{\mathrm{D}}-10.2(c$ 1.43, $21^{\circ} \mathrm{C}, \mathrm{CHCl}_{3}$ ); IR 2956 (s), 2876 (m), 1740 (s), 1375 (m), 1243 (s); ${ }^{1} \mathrm{H}$ NMR (500 MHz, $\left.\mathrm{CDCl}_{3}\right) \delta 4.97(\psi \mathrm{q}, J=6.5 \mathrm{~Hz}, 1 \mathrm{H}), 3.64(\mathrm{t}, J=7.0 \mathrm{~Hz}, 2 \mathrm{H}), 2.04(\mathrm{~s}, 3 \mathrm{H}), 1.79(\mathrm{q}, J=6.5 \mathrm{~Hz}$, 2H), $1.56(\mathrm{~m}, 2 \mathrm{H}), 1.30(\mathrm{bm}, 6 \mathrm{H}), 0.96(\mathrm{t}, J=8.0 \mathrm{~Hz}, 9 \mathrm{H}), 0.88(\mathrm{t}, J=6.5 \mathrm{~Hz}, 2 \mathrm{H}), 0.59(\mathrm{q}, J=$ $8.0 \mathrm{~Hz}, 6 \mathrm{H}) ;{ }^{13} \mathrm{C}\left(125.8 \mathrm{MHz}, \mathrm{CDCl}_{3}\right) \delta 170.9,72.1,59.6,37.4,34.5,31.9,25.0,22.8,21.5,14.2$, 7.0, 4.5; HRMS $m / z\left(E S I, M+\mathrm{Na}^{+}\right)$calcd 325.2175, found 325.2154.

\section{(+)-Silyl-enol-ether-alcohol (31):}

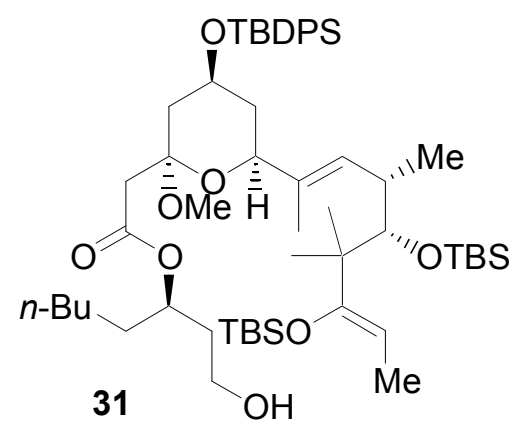


$n$-BuLi $(2.5 \mathrm{M}$ in hexanes, $111 \mu \mathrm{L}, 0.278 \mathrm{mmol})$ was added to a cold $\left(-10^{\circ} \mathrm{C}\right)$ solution of $i \operatorname{Pr}_{2} \mathrm{NH}(38.5 \mu \mathrm{L}, 0.27 \mathrm{mmol})$ in THF $(1.8 \mathrm{~mL})$ the solution was stirred for 15 minutes, then cooled to $-78{ }^{\circ} \mathrm{C}$. A solution of $\mathbf{3 0}(78.5 \mathrm{mg}, 0.259 \mathrm{mmole})$ in THF $(0.9 \mathrm{~mL})$ was added dropwise and the reaction stirred for 10 minutes at $-78^{\circ} \mathrm{C}$ and 3 minutes at $-42{ }^{\circ} \mathrm{C}$, a solution of 29 (80 $\mathrm{mg}, 0.10 \mathrm{mmole})$ in THF $(1.8 \mathrm{~mL})$ was added dropwise and the reaction stirred for 2 hours at $-42{ }^{\circ} \mathrm{C}$. The reaction was quenched by the addition of water and the product extracted with diethyl ether, the combined organics were washed with brine and dried over magnesium sulfate. The crude material was concentrated and passed through a plug of silica gel (load with hexanes flush with 30:1 hexanes:diethyl ether), the eluent was collected, concentrated and placed in a flask with methanol $(15 \mathrm{~mL})$ along with citric acid (46 $\mathrm{mg}, 0.22 \mathrm{mmole})$. The suspension was heated to $65{ }^{\circ} \mathrm{C}$ and stirred for 35 minutes (at which point the solution became homogeneous). The solvent was removed in vacuo and the residue purified by chromatography (hexanes $\rightarrow$ 7:1 hexanes:diethyl ether) to give $76 \mathrm{mg}$ (75\% over two steps) of $\mathbf{3 1}$ as a clear oil. $[\alpha]_{\mathrm{D}}+3.8\left(\mathrm{c} 1.0,22^{\circ} \mathrm{C}, \mathrm{CHCl}_{3}\right) ; \mathrm{IR} 3842(\mathrm{bm}), 3073(\mathrm{w}), 2957(\mathrm{~s}), 2858(\mathrm{~s}), 1733(\mathrm{~m}), 1664(\mathrm{~m})$, 1473 (m), 1381 (m), 1318 (m), 1254 (s), 1112 (s), 1041 (s), 837 (s), 774 (s), 736 (s), 702 (s); ${ }^{1} \mathrm{H}$ NMR (500 MHz, $\left.\mathrm{CDCl}_{3}\right) \delta 7.66(\mathrm{~m}, 4 \mathrm{H}), 7.38(\mathrm{~m}, 6 \mathrm{H}), 5.58(\mathrm{~d}, J=9.5 \mathrm{~Hz}, 1 \mathrm{H}), 5.04(\mathrm{~m}, 1 \mathrm{H})$, $4.52(\mathrm{q}, J=7.0 \mathrm{~Hz}, 1 \mathrm{H}), 4.14(\mathrm{tt}, J=11.0,4.5 \mathrm{~Hz}, 1 \mathrm{H}), 3.81(\mathrm{~s}, 1 \mathrm{H}), 3.57(\mathrm{~m}, 3 \mathrm{H}), 3.10(\mathrm{~s}, 3 \mathrm{H})$, $2.81(\mathrm{~d}, J=13.5 \mathrm{~Hz}, 1 \mathrm{H}), 2.60(\mathrm{dd}, J=9.0,7.5 \mathrm{~Hz}, 1 \mathrm{H}), 2.52(\mathrm{~d}, J=13.5 \mathrm{~Hz}, 1 \mathrm{H}), 2.45(\mathrm{dd}, J=$ 8.0, $5.0 \mathrm{~Hz}, 1 \mathrm{H}), 2.25(\mathrm{dd}, J=13.0,3.5 \mathrm{~Hz}, 1 \mathrm{H}), 1.85(\mathrm{~m}, 1 \mathrm{H}), 1.64(\mathrm{~m}, 4 \mathrm{H}), 1.53(\mathrm{~d}, J=7.0 \mathrm{~Hz}$ 3H), 1.49 (s, 3H), $1.30(\mathrm{bm}, 6 \mathrm{H}), 1.05(\mathrm{~s}, 9 \mathrm{H}), 1.01(\mathrm{~s}, 9 \mathrm{H}), 1.00(\mathrm{~s}, 3 \mathrm{H}), 0.92(\mathrm{~s}, 9 \mathrm{H}), 0.89(\mathrm{~m}$, 6H), 0.84 (s, 3H), 0.21 (s, 3H), $0.20(\mathrm{~s}, 3 \mathrm{H}), 0.10(\mathrm{~s}, 3 \mathrm{H}), 0.06(\mathrm{~s}, 3 \mathrm{H}) ;{ }^{13} \mathrm{C}\left(125.8 \mathrm{MHz}, \mathrm{CDCl}_{3}\right)$ $\delta 170.5,157.5,136.0,135.9,134.8,134.6,130.9,130.1,129.7,127.7,127.7,99.6,99.0,80.9$, $74.4,72.1,66.9,58.7,47.8,46.4,43.1,42.8,38.7,37.6,34.7,33.9,31.8,27.2,26.8,26.6,25.3$, 
$22.9,22.7,21.6,19.4,19.3,14.4,14.2,12.8,12.0,-2.4,-2.5,-2.9,-3.2$; HRMS $m / z$ (ESI, $\mathrm{M}+\mathrm{Na}^{+}$) calcd 1003.6305, found 1003.6304.

(+)-4-Triethylsilyoxylnonene (57):

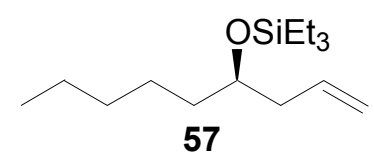

To a cold $\left(0^{\circ} \mathrm{C}\right)$ solution of $\mathbf{5 6}(47 \mathrm{mg}, 0.33 \mathrm{mmol})$ in DMF $(1 \mathrm{~mL})$ was added imidazole (112 $\mathrm{mg}, 1.6 \mathrm{mmol})$ and chlorotriethylsilane $(221 \mu \mathrm{l}, 1.3 \mathrm{mmol})$, the mixture was warmed to room temperature and stirred for $4 \mathrm{~h}$. The reaction was quenched with water and extracted with diethyl ether. The combined organic extracts were washed with brine, dried over magnesium sulfate, filtered, concentrated (rt, $60 \mathrm{mmHg}$ ) and purified by chromatography (hexanes) to give 57 as a clear oil $(68 \mathrm{mg}, 80 \%) . \quad[\alpha]_{\mathrm{D}}+11.4\left(c 0.78,22^{\circ} \mathrm{C}, \mathrm{CHCl}_{3}\right)$; IR $2956(\mathrm{~s}), 2876(\mathrm{~s}), 1458$ (m), $1239(\mathrm{~m}), 1005(\mathrm{~m}), 911(\mathrm{~m}), 724(\mathrm{~s}) ;{ }^{1} \mathrm{H}$ NMR $\left(500 \mathrm{MHz}, \mathrm{CDCl}_{3}\right) \delta 5.83\left(\mathrm{ddt}, J_{\mathrm{t}}=7.0, J_{\mathrm{d}}=\right.$ 17.5, $10.0 \mathrm{~Hz}, 1 \mathrm{H}), 5.04(\mathrm{~m}, 2 \mathrm{H}), 3.69$ (quint, $J=6.0,1 \mathrm{H}), 2.22(\mathrm{~m}, 2 \mathrm{H}), 1.50-1.25(\mathrm{bm}, 8 \mathrm{H})$, $0.97(\mathrm{t}, J=7.5,9 \mathrm{H}), 0.89(\mathrm{t}, J=7.0,3 \mathrm{H}), 0.61(\mathrm{q}, J=7.5,6 \mathrm{H}) ;{ }^{13} \mathrm{C}\left(125.8 \mathrm{MHz}, \mathrm{CDCl}_{3}\right) \delta$ 135.6, 116.8, 72.3, 42.3, 37.1, 32.2, 25.3, 22.9, 14.3, 7.2, 5.2; HRMS $m / z\left(E S I, M+\mathrm{Na}^{+}\right)$calcd 279.2115, found 279.2106.

\section{(-)-3-(triethylsilyloxy)-octanal (24):}

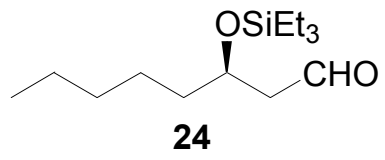


Ozone was bubbled through a cold $\left(-78^{\circ} \mathrm{C}\right)$ solution of $57(48 \mathrm{mg}, 0.18 \mathrm{mmol})$ in DCM (4 mL) until a pale blue color was observed, the solution was degassed with argon, and triphenylphosphine $(\sim 400 \mathrm{mg})$ in DCM $(\sim 1 \mathrm{~g} / \mathrm{mL})$ was added. The solution was allowed to warm to $-15{ }^{\circ} \mathrm{C}$ over two hours, concentrated and purified by silica gel chromatography (50:1 hexanes/DCM $\rightarrow 5: 1$ hexanes/diethyl ether) to give 24 as a colorless oil $(46 \mathrm{mg}, 96 \%)$. [ $\alpha]_{\mathrm{D}}-7.9$ (c 0.43, $21{ }^{\circ} \mathrm{C}, \mathrm{CHCl}_{3}$ ); IR 2956 (s), 2877 (s), 1727 (s), 1457 (w), 1379 (w), 1240 (w), 1102 (m), 1005 (m); ${ }^{1} \mathrm{H}$ NMR (500 MHz, $\left.\mathrm{CDCl}_{3}\right) \delta 9.82(\mathrm{t}, J=2.5 \mathrm{~Hz}, 1 \mathrm{H}), 4.20(\mathrm{q}, J=6.0 \mathrm{~Hz}, 1 \mathrm{H}), 2.53$ (m, 2H), 1.60-1.49 (bm, 2H), 1.37-1.25 (bm, 6H), $0.96(\mathrm{t}, J=8.0 \mathrm{~Hz}, 9 \mathrm{H}), 0.90$ (t, $J=7.0 \mathrm{~Hz}$, $3 \mathrm{H}), 0.61(\mathrm{q}, J=8.0 \mathrm{~Hz}, 6 \mathrm{H}) ;{ }^{13} \mathrm{C}\left(125.8 \mathrm{MHz}, \mathrm{CDCl}_{3}\right) \delta 202.7,68.4,51.1,38.2,32.0,25.1$, 22.8, 14.2, 7.0, 5.2; HRMS $m / z$ (ESI, $\mathrm{M}+\mathrm{Na}^{+}$) calcd 281.1907, found 281.1898.

\section{a-Bromo-ketone (36):}

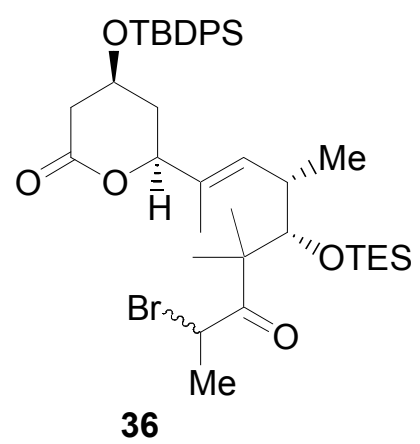

To a cooled $\left(0{ }^{\circ} \mathrm{C}\right)$ solution of $23(800 \mathrm{mg}, 1.03 \mathrm{mmol})$ in THF $(45 \mathrm{~mL})$ was added recrystallized N-Bromosuccinimide (NBS) (201 mg, $1.1 \mathrm{mmol})$ and the reaction was stirred for 45 minutes then quenched with sodium bicarbonate. The solution was poured in to a mixture of brine and saturated aqueous sodium bicarbonate and the product extracted with ethyl acetate. The combined organic layers were dried over magnesium sulfate, filtered, concentrated and purified by chromatography (hexanes $\rightarrow$ 10:1 hexanes/ethyl acetate $\rightarrow$ 6:1 hexanes/ethyl acetate) 
to give $765 \mathrm{mg}(100 \%)$ of $\mathbf{3 6}$ as a 3:1 mixture of diastereomers (spectral data for major diastereomer only). IR 3473 (bw), 3072 (w), 2959 (s), 2877 (s), 1742 (s), 1711 (m), 1472 (m), 1428 (m), 1233 (s), 1113 (s), 1009 (s) 703 (s); ${ }^{1} \mathrm{H}$ NMR (500 MHz, CDCl 3 ) $\delta 7.65$ (m, 4H), 7.46 (m, 2H), $7.40(\psi \mathrm{t}, J=7.0 \mathrm{~Hz}, 4 \mathrm{H}), 5.60(\mathrm{~d}, J=9.5 \mathrm{~Hz}, 1 \mathrm{H}), 4.72$ (q, $J=6.5 \mathrm{~Hz}, 1 \mathrm{H}), 4.30(\mathrm{dd}, J$

$=11.5,3.0 \mathrm{~Hz}, 1 \mathrm{H}), 4.12(\mathrm{~m}, 1 \mathrm{H}), 3.40(\mathrm{~d}, J=1.0 \mathrm{~Hz}, 1 \mathrm{H}), 2.71(\mathrm{dd}, J=17.5,6.0 \mathrm{~Hz}, 1 \mathrm{H}), 2.49$ (dd, $J=17.5,8.0 \mathrm{~Hz}, 1 \mathrm{H}), 2.38$ (apparent quintet, $J=7.5 \mathrm{~Hz}, 1 \mathrm{H}), 1.90(\mathrm{~m}, 1 \mathrm{H}), 1.83(\mathrm{~m}, 1 \mathrm{H})$, $1.68(\mathrm{~d}, J=6.5 \mathrm{~Hz}, 3 \mathrm{H}), 1.56(\mathrm{~s}, 3 \mathrm{H}), 1.22(\mathrm{~s}, 3 \mathrm{H}), 1.17(\mathrm{~s}, 3 \mathrm{H}), 1.07$ (s, 9H), $1.00(\mathrm{~m}, 9 \mathrm{H}), 0.93$ $(\mathrm{d}, J=7.5 \mathrm{~Hz}, 3 \mathrm{H}), 0.68(\mathrm{~m}, 6 \mathrm{H}) ;{ }^{13} \mathrm{C}\left(125.8 \mathrm{MHz}, \mathrm{CDCl}_{3}\right) \delta 209.5,170.9,135.8,133.6,133.3$, 131.0, 130.4, 130.3, 130.2, 128.1, 128.0, 124.9, 82.2, 81.5, 65.6, 54.2, 41.4, 40.0, 37.3, 35.4, 27.0, 25.4, 21.3, 21.1, 19.2, 11.9, 7.4, 7.4, 5.8, 5.8; HRMS $m / z$ (ESI, $\left.\mathrm{M}+\mathrm{Na}^{+}\right)$calcd 765.2977, found 765.2975 .

\section{$\beta$-Hydroxy ketone (58):}

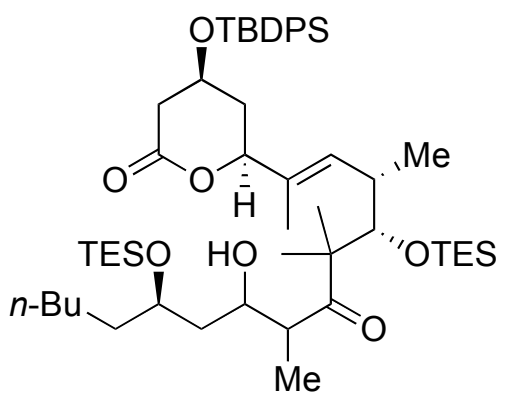

58

IR 3503 (bm), 2957 (s), 2876 (s), 1744 (s), 1700 (s), 1462 (s), 1379 (s), 1235 (s), 1112 (s), 1009 (s), 739 (s), 702 (s); HRMS $m / z\left(E S I, M+\mathrm{Na}^{+}\right.$) calcd 945.5887, found 945.5880.

\section{(-)-ß-Hydroxy-enone (59):}




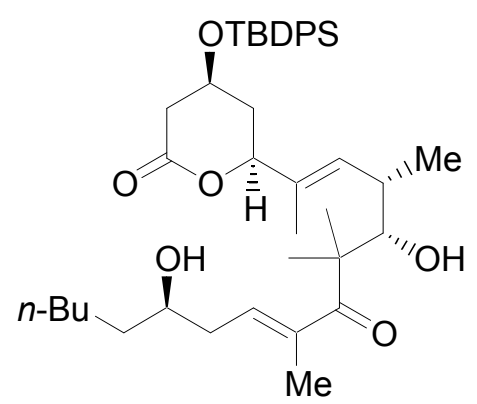

59

A solution of $\mathrm{HF}$ (49\% aqueous, $800 \mu \mathrm{L}, 24.4 \mathrm{mmol})$ in $\mathrm{MeCN}(7.2 \mathrm{~mL}$ ) was added to 39 (88 $\mathrm{mg}, 97 \mu \mathrm{mol}$ ) in a plastic vial. After 4 minutes the vial was rinsed (diethyl ether) into a separation funnel containing aqueous sodium bicarbonate. The two phases were separated and the aqueous phase was extracted twice with ethyl acetate, the combined organic layers were washed with sodium bicarbonate and brine, dried over sodium sulfate, filtered, concentrated and purified by silica gel chromatography ( $8: 1$ hexanes:ethyl acetate $\rightarrow 3: 1$ hexanes:ethyl acetate) to

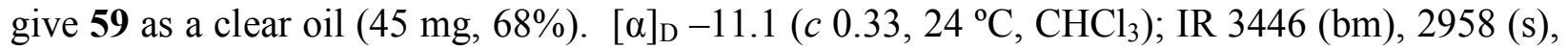
2930 (s), 2858 (s), 1734 (s), 1653 (w), 1472 (m), 1428 (m), 1379 (m), 1240 (m), 1112 (s); ${ }^{1} \mathrm{H}$ $\operatorname{NMR}\left(500 \mathrm{MHz}, \mathrm{CDCl}_{3}\right) \delta 7.64$ (apparent dd, $\left.J=8.0,1.5 \mathrm{~Hz}, 4 \mathrm{H}\right), 7.47$ (m, 2H), 7.41 (apparent $\left.\mathrm{dt}, J_{\mathrm{t}}=7.5, J_{\mathrm{d}}=1.5 \mathrm{~Hz}, 4 \mathrm{H}\right), 5.97\left(\mathrm{dt}, J_{\mathrm{t}}=7.0, J_{\mathrm{d}}=1.5 \mathrm{~Hz}, 1 \mathrm{H}\right), 5.44(\mathrm{~d}, J=10.5 \mathrm{~Hz}, 1 \mathrm{H}), 4.34$ $(\mathrm{dd}, J=12.0,3.5 \mathrm{~Hz}, 1 \mathrm{H}), 4.11(\mathrm{~m}, 1 \mathrm{H}), 3.85(\mathrm{~d}, J=7.5 \mathrm{~Hz}, 1 \mathrm{H}), 3.71(\mathrm{~m}, 1 \mathrm{H}), 3.56(\mathrm{dd}, J=$ 7.5, $2.5 \mathrm{~Hz}, 1 \mathrm{H}), 2.67(\mathrm{~m}, 2 \mathrm{H}), 2.47(\mathrm{dd}, J=17.5,8.0 \mathrm{~Hz}, 1 \mathrm{H}), 2.30(\mathrm{~m}, 1 \mathrm{H}), 2.23(\mathrm{~m}, 1 \mathrm{H}), 2.13$ $(\mathrm{d}, J=5.0 \mathrm{~Hz}, 1 \mathrm{H}), 1.90(\mathrm{~m}, 1 \mathrm{H}), 1.84(\mathrm{~m}, 1 \mathrm{H}), 1.78(\mathrm{~s}, 3 \mathrm{H}), 1.62(\mathrm{~d}, J=1.0 \mathrm{~Hz}, 3 \mathrm{H}), 1.45(\mathrm{~m}$, 3H), $1.30(\mathrm{bm}, 8 \mathrm{H}), 1.20(\mathrm{~s}, 3 \mathrm{H}), 1.06(\mathrm{~s}, 9 \mathrm{H}), 1.02(\mathrm{~d}, J=7.0 \mathrm{~Hz}, 3 \mathrm{H}), 0.89(\mathrm{t}, J=7.0 \mathrm{~Hz}, 3 \mathrm{H})$; ${ }^{13} \mathrm{C}\left(125.8 \mathrm{MHz}, \mathrm{CDCl}_{3}\right) \delta 216.0,171.2,138.0,135.8,135.8,133.5,133.3,132.3,131.2,131.2$ $130.3,130.3,128.1,128.1,82.8,77.4,71.0,65.5,50.2,40.0,37.6,37.4,36.1,34.4,32.1,27.0$, 25.9, 25.6, 22.8, 22.8, 19.9, 19.2, 15.0, 14.3, 11.3; HRMS m/z (ESI, $\left.\mathrm{M}+\mathrm{Na}^{+}\right)$calcd 699.4051, found 699.4055 . 


\section{(-)-Triol (40):}

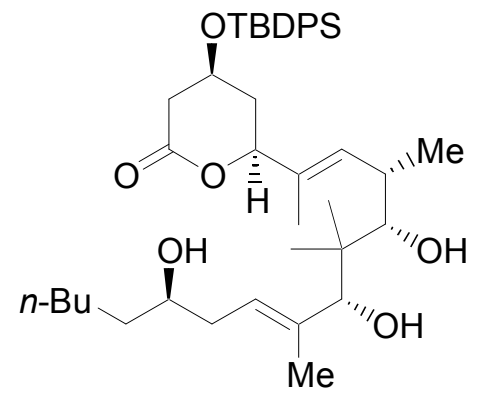

40

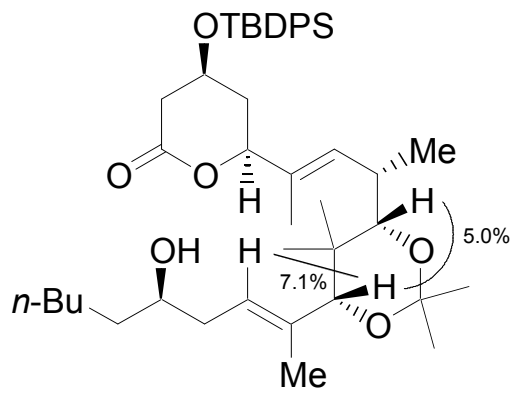

41

To a cold $\left(-10^{\circ} \mathrm{C}\right)$ solution of $59(140 \mathrm{mg}, 0.207 \mathrm{mmol})$ in THF $(14 \mathrm{~mL})$ was added catecholborane $(442 \mu \mathrm{L}, 4.13 \mathrm{mmol})$ and the reaction was stirred at $-10^{\circ} \mathrm{C}$ for $6 \mathrm{~h}$. Methanol (4 $\mathrm{mL}$ ) was carefully added to the reaction, followed by saturated aqueous potassium sodium tartrate $(4 \mathrm{~mL})$, and pinacol $(300 \mathrm{mg})$. The reaction was warmed to room temperature and stirred overnight. The solution was diluted with ethyl acetate and then washed with $0.5 \mathrm{M} \mathrm{NaOH}$ until the aqueous layer was colorless and then once with brine. The organic layer was dried over sodium sulfate, filtered, concentrated and purified by chromatography (4:1 hexanes/ethyl acetate $\rightarrow 1: 1$ hexanes/ethyl acetate) to give $122 \mathrm{mg}(87 \%)$ of $\mathbf{4 0}$ as a clear oil. Stereochemistry of the diol, and olefin geometry was confirmed by nOe analysis of the acetonide (41). 40 (3.1 $\mathrm{mg}, 4.6$ $\mu$ mole) was reacted with 2,2-dimethoxypropane $(2 \mathrm{eq})$ in acetone $(0.01 \mathrm{M})$ and catalytic CSA (0.2 eq) to give 41 (3.1 mg, 95\%) after chromatography (hexanes $\rightarrow$ 3:1 hexane/ethyl acetate). 40: $[\alpha]_{\mathrm{D}}-4.8\left(c 0.18,22{ }^{\circ} \mathrm{C}, \mathrm{CHCl}_{3}\right)$; IR $3386(\mathrm{bm}), 2958$ (s), 2930 (s), 2858 (s), 1734 (s), 1428 (s), 1379 (s), 1260 (m), 1112 (s), 1011 (s), 823 (w); ${ }^{1} \mathrm{H}$ NMR (500 MHz, CDCl 3 ) $\delta 7.65$ (apparent dd, $J=8.0,1.5 \mathrm{~Hz}, 4 \mathrm{H}) ; 7.46(\mathrm{~m}, 2 \mathrm{H}), 7.40(\mathrm{~m}, 4 \mathrm{H}), 5.70(\mathrm{~d}, J=10.0 \mathrm{~Hz}, 1 \mathrm{H}), 5.47$ (t, $J=7.5 \mathrm{~Hz}, 1 \mathrm{H}), 4.38(\mathrm{dd}, J=12.0,3.0 \mathrm{~Hz}, 1 \mathrm{H}), 4.12(\mathrm{~m}, 1 \mathrm{H}), 3.98(\mathrm{~s}, 1 \mathrm{H}), 3.67(\mathrm{bs}, 1 \mathrm{H})$, $3.55(\mathrm{~s}, 1 \mathrm{H}), 3.52(\mathrm{bs}, 1 \mathrm{H}), 2.68(\mathrm{~m}, 2 \mathrm{H}), 2.48(\mathrm{dd}, J=17.0,8.5 \mathrm{~Hz}, 1 \mathrm{H}), 2.22(\mathrm{t}, J=7.0 \mathrm{~Hz}$ 
$2 \mathrm{H}), 1.94\left(\mathrm{dt}, J_{\mathrm{t}}=3.5, J_{\mathrm{d}}=13.0 \mathrm{~Hz}, 1 \mathrm{H}\right), 1.87(\mathrm{~m}, 1 \mathrm{H}), 1.68(\mathrm{~s}, 3 \mathrm{H}), 1.64(\mathrm{~d}, J=1.0 \mathrm{~Hz}, 3 \mathrm{H})$, 1.49-1.44 (bm, 3H), 1.36-1.26 (bm, 6H), 1.07 (s, 9H), 1.03 (d, $J=7.0 \mathrm{~Hz}, 3 \mathrm{H}), 0.90$ (t, $J=7.0$ $\mathrm{Hz}, 3 \mathrm{H}), 0.88(\mathrm{~s}, 3 \mathrm{H}), 0.71(\mathrm{~s}, 3 \mathrm{H}) ;{ }^{13} \mathrm{C}\left(125.8 \mathrm{MHz}, \mathrm{CDCl}_{3}\right) \delta 171.1,138.5,135.9,135.8,133.6$, $133.4,131.5,130.3,130.2,130.0,128.1,128.0,126.0,87.3,83.8,82.6,71.9,65.7,42.6,40.1$, 37.4, 37.4, 35.9, 34.1, 32.1, 27.0, 25.6, 22.8, 20.3, 19.2, 15.9, 15.3, 14.3, 11.5; HRMS $m / z$ (ESI, $\mathrm{M}+\mathrm{Na}^{+}$) calcd 701.4208, found 701.4212.

41: $[\alpha]_{\mathrm{D}}-16.5\left(c 0.5,24{ }^{\circ} \mathrm{C}, \mathrm{CHCl}_{3}\right)$; IR 3447 (bm), 2959 (s), 2931(s), 2859 (s), 1741 (s), 1465 (m), 1428 (m), 1378 (s), 1254 (s), 1168 (m), 1112 (s), 702 (s); ${ }^{1} \mathrm{H}$ NMR (500 MHz, $\left.\mathrm{CDCl}_{3}\right) \delta$ $7.65(\mathrm{~m}, 4 \mathrm{H}), 7.46$ (apparent t, $J=7.0 \mathrm{~Hz}, 2 \mathrm{H}), 7.40(\mathrm{~m}, 4 \mathrm{H}), 5.71(\mathrm{~d}, J=9.5 \mathrm{~Hz}, 1 \mathrm{H}), 5.38$ (nOe 7.1\%) (t, $J=7.0 \mathrm{~Hz}, 1 \mathrm{H}), 4.37(\mathrm{dd}, J=12.5,3.0 \mathrm{~Hz}, 1 \mathrm{H}), 4.12(\mathrm{~m}, 1 \mathrm{H}), 3.91(\mathrm{nOe} 7.1 \%$, $5.0 \%)(\mathrm{s}, 1 \mathrm{H}), 3.66(\mathrm{~m}, 1 \mathrm{H}), 3.43(\mathrm{nOe} 5.0 \%)(\mathrm{s}, 1 \mathrm{H}), 2.68(\mathrm{~m}, 2 \mathrm{H}), 2.49(\mathrm{dd}, J=17.0,8.0 \mathrm{~Hz}$, $1 \mathrm{H}), 2.23(\mathrm{~m}, 2 \mathrm{H}), 1.94\left(\mathrm{dt}, J_{\mathrm{t}}=3.5, J_{\mathrm{d}}=13.0 \mathrm{~Hz}, 1 \mathrm{H}\right), 1.86(\mathrm{~m}, 1 \mathrm{H}), 1.66(\mathrm{~s}, 3 \mathrm{H}), 1.63(\mathrm{~d}, J=$ $1.0 \mathrm{~Hz}, 3 \mathrm{H}), 1.46(\mathrm{~m}, 3 \mathrm{H}), 1.44$ (s, 3H), 1.43 (s, 3H), 1.32-1.26 (m, 6H), 1.07 (s, 9H), 0.97 (d, $J$ $=7.0 \mathrm{~Hz}, 3 \mathrm{H}), 0.90(\mathrm{t}, J=7.0 \mathrm{~Hz}, 3 \mathrm{H}), 0.76(\mathrm{~s}, 3 \mathrm{H}), 0.74(\mathrm{~s}, 3 \mathrm{H}) ;{ }^{13} \mathrm{C}\left(125.8 \mathrm{MHz}, \mathrm{CDCl}_{3}\right) \delta$ $171.2,135.9,135.8,135.4,133.6,133.4,132.7,130.3,130.2,129.1,128.1,128.0,126.0,98.8$ $84.3,82.6,82.0,71.9,65.7,40.1,38.3,37.3,37.2,36.2,32.1,31.8,31.8,30.2,27.0,25.6,22.8$, 22.2, 20.7, 19.4, 19.2, 15.6, 15.3, 14.3, 11.6; HRMS $m / z\left(\mathrm{ESI}, \mathrm{M}+\mathrm{Na}^{+}\right)$calcd 741.4521, found 741.4504 .

\section{Seco Acid (42):}




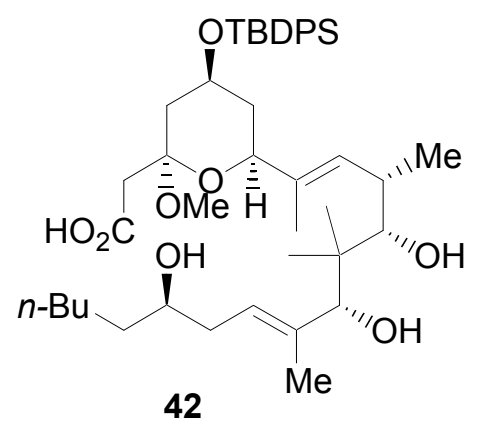

$n$-BuLi $(2.5 \mathrm{M}$ in hexanes, $359 \mu \mathrm{L}, 0.90 \mathrm{mmol})$ was added to a cold $\left(-10^{\circ} \mathrm{C}\right)$ solution of $i \operatorname{Pr}_{2} \mathrm{NH}(126 \mu \mathrm{L}, 0.90 \mathrm{mmol})$ in THF $(8 \mathrm{~mL})$ the solution was stirred for 15 minutes, then cooled to $-78{ }^{\circ} \mathrm{C}$. A solution of ethyl acetate $(88 \mu \mathrm{L}, 0.90 \mathrm{mmole})$ in THF $(3 \mathrm{~mL})$ was added dropwise and the reaction stirred for 5 minutes at $-78{ }^{\circ} \mathrm{C} . \quad \mathbf{4 0}(61 \mathrm{mg}, 0.090 \mathrm{mmole})$ was added as a solution in THF ( $5 \mathrm{~mL}+3 \mathrm{~mL}$ rinse) over 2 minutes and the reaction was warmed to $-42{ }^{\circ} \mathrm{C}$ and stirred for 1 hour. $0.1 \mathrm{M} \mathrm{NaHSO}_{4}$ was added to stop the reaction and the product was extracted with ethyl acetate, the combined organics were washed with brine, dried over sodium sulfate, and concentrated to leave a pale yellow oil. The residue was dissolved in methanol ( $28 \mathrm{~mL})$, placed in a sealed tube along with $60 \mathrm{mg}(0.28 \mathrm{mmole})$ of citric acid and heated to $70{ }^{\circ} \mathrm{C}$ for 2 hours. The reaction was cooled to room temperature, diluted with ethyl acetate, washed with sodium bicarbonate and brine, dried over sodium sulfate, and concentrated. Upon TLC examination two spots were observable, the major was ethyl acetate (and methanol) addition to lactone, and the minor was the same but with an acetate protection at $\mathrm{C} 17$. Both spots were isolated by silica gel chromatography (4:1 hexanes:ethyl acetate $\rightarrow$ 1:1 hexanes:ethyl acetate) and then recombined. The combined products were dissolved in water/methanol/THF $(1.55 \mathrm{~mL}$, $3.1 \mathrm{~mL}, 3.1 \mathrm{~mL})$ and $\mathrm{LiOH}(65 \mathrm{mg}, 1.55 \mathrm{mmole})$ was added and the solution stirred overnight. Ethyl acetate was added and two phases were separated. The organic phase was washed with saturated aqueous sodium bicarbonate and brine then dried over sodium sulfate and concentrated. This gave 42 (43.3 mg, 64\% over 3 steps) in $>90 \%$ purity (by ${ }^{1} \mathrm{H}$ NMR). ${ }^{1} \mathrm{H}$ NMR (500 MHz, 
$\left.\mathrm{CDCl}_{3}\right) \delta 7.66(\mathrm{~m}, 4 \mathrm{H}), 7.43(\mathrm{~m}, 2 \mathrm{H}), 7.38(\mathrm{~m}, 4 \mathrm{H}), 5.66(\mathrm{~d}, J=9.0 \mathrm{~Hz}, 1 \mathrm{H}), 5.48(\mathrm{t}, J=7.0 \mathrm{~Hz}$ 1H), $4.13(\mathrm{~m}, 1 \mathrm{H}), 3.99(\mathrm{~s}, 1 \mathrm{H}), 3.85(\mathrm{~d}, J=11.5 \mathrm{~Hz}, 1 \mathrm{H}), 3.67(\mathrm{~m}, 1 \mathrm{H}), 3.53(\mathrm{~s}, 1 \mathrm{H}), 2.79(\mathrm{~d}, J$ $=15.0 \mathrm{~Hz}, 1 \mathrm{H}), 2.69(\mathrm{~m}, 1 \mathrm{H}), 2.52(\mathrm{~d}, J=15.0 \mathrm{~Hz}, 1 \mathrm{H}), 2.24-2.17(\mathrm{~m}, 3 \mathrm{H}), 2.08-2.02(\mathrm{~m}, 2 \mathrm{H})$, 1.70-1.68 (m, 2H), $1.68(\mathrm{~s}, 3 \mathrm{H}), 1.64(\mathrm{~m}, 1 \mathrm{H}), 1.58(\mathrm{~s}, 3 \mathrm{H}), 1.50-1.42(\mathrm{~m}, 4 \mathrm{H}), 1.34-1.24(\mathrm{~m}$, 8H), 1.05 (s, 9H), 1.02 (d, $J=7.0 \mathrm{~Hz}, 3 \mathrm{H}), 0.90(\mathrm{t}, J=6.5 \mathrm{~Hz}, 3 \mathrm{H}), 0.88(\mathrm{~s}, 3 \mathrm{H}), 0.72(\mathrm{~s}, 3 \mathrm{H})$; HRMS $m / z$ (ESI, $\mathrm{M}+\mathrm{Na}^{+}$) calcd 775.4578, found 775.4606.

\section{Ene-ester (44):}

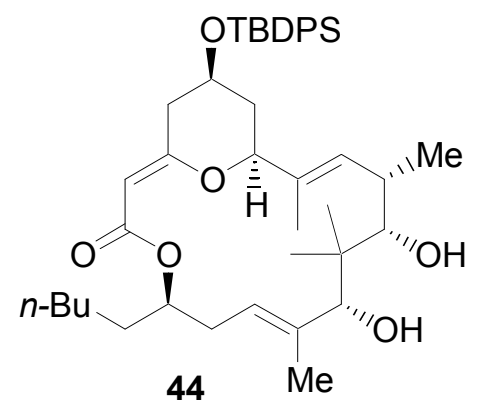

This procedure represents the highest yield obtained by this method, typically reactions were run on a $28 \mathrm{mg}$ scale and gave 5-11 $\mathrm{mg}$ (20-42\%) of the products. 42 (6.4 mg, 8.5 Hmole) was loaded into a $10 \mathrm{~mL}$ round-bottomed flask and azeotroped twice with anhydrous toluene. A stock solution was prepared by dissolving triethylamine (46 $\mu \mathrm{L}, 0.33$ mmole) and 2,4,6trichlorobenzoyl chloride $(46 \mu \mathrm{L}, 0.30 \mathrm{mmole})$ in THF $(8.3 \mathrm{~mL})$. A portion $(714 \mu \mathrm{L})$ of the stock solution was added to the flask containing 42. The solution was stirred at room temperature for 15 hours -if after that time the solution was 'cloudy' the yields were generally low, if the solution was clear with a noticeable amount of white precipitate the yields were generally higher- then filtered through a cotton stuffed pipette and concentrated. The product azeotroped twice with anhydrous toluene. The yellow residue was then dissolved in anhydrous toluene $(3 \mathrm{~mL})$ and added over 7 hours via syringe pump to a solution of refluxing DMAP (6.2 
$\mathrm{mg}, 0.051 \mathrm{mmole})$ in toluene $(8.4 \mathrm{~mL})$. After addition was complete the suspension was stirred for an additional 30 minutes and then cooled to room temperature, diluted with ethyl acetate, washed with $0.1 \mathrm{M} \mathrm{NaHSO}_{4}$ and brine, dried over magnesium sulfate, filtered and concentrated. Purification by silica gel chromatography (hexanes $\rightarrow$ 10:1 hexanes:ethyl acetate $\rightarrow 6: 1$ hexanes:ethyl acetate) provided a mixture of $\mathbf{4 4}$ and $\mathbf{4 3}\left(4.2 \mathrm{mg}\right.$ total, 82:18 44:43, 70\%). ${ }^{1} \mathrm{H}$ NMR (500 MHz, $\left.\mathrm{CDCl}_{3}\right) \delta 7.67(\mathrm{~m}, 2 \mathrm{H}), 7.43(\mathrm{~m}, 2 \mathrm{H}), 7.38(\mathrm{~m}, 4 \mathrm{H}), 5.53(\mathrm{~d}, J=10.0 \mathrm{~Hz}, 1 \mathrm{H})$, $5.25(\mathrm{dd}, J=8.5,4.0 \mathrm{~Hz}, 1 \mathrm{H}), 4.82(\mathrm{~m}, 1 \mathrm{H}), 4.64(\mathrm{~s}, 1 \mathrm{H}), 4.59(\mathrm{t}, J=8.5 \mathrm{~Hz}, 1 \mathrm{H}), 4.15(\mathrm{~d}, J=$ $11.5 \mathrm{~Hz}, 1 \mathrm{H}), 3.95(\mathrm{~s}, 1 \mathrm{H}), 3.48(\mathrm{~s}, 1 \mathrm{H}), 3.13(\mathrm{~d}, J=15.0 \mathrm{~Hz}, 1 \mathrm{H}), 2.91(\mathrm{~s}, 1 \mathrm{H}), 2.87(\mathrm{~m}, 1 \mathrm{H})$, $2.31(\mathrm{~m}, 1 \mathrm{H}), 2.11(\mathrm{~m}, 1 \mathrm{H}), 1.94(\mathrm{~m}, 1 \mathrm{H}), 1.80(\mathrm{dd}, J=13.0,7.0 \mathrm{~Hz}, 1 \mathrm{H}), 1.71(\mathrm{~s}, 1 \mathrm{H}), 1.65(\mathrm{~s}$ $3 \mathrm{H}), 1.64(\mathrm{~d}, J=1.5 \mathrm{~Hz}, 3 \mathrm{H}), 1.64(\mathrm{~d}, J=1.5 \mathrm{~Hz}, 3 \mathrm{H}), 1.50-1.40(\mathrm{~m}, 2 \mathrm{H}), 1.32-1.22(\mathrm{~m}, 6 \mathrm{H})$, $1.07(\mathrm{~s}, 9 \mathrm{H}), 1.00(\mathrm{t}, J=7.0 \mathrm{~Hz}, 3 \mathrm{H}), 1.00(\mathrm{~s}, 3 \mathrm{H}), 0.87(\mathrm{t}, J=7.0 \mathrm{~Hz}, 3 \mathrm{H}), 0.72(\mathrm{~s}, 3 \mathrm{H})$. HRMS $m / z$ (ESI, $\mathrm{M}+\mathrm{Na}^{+}$) calcd 725.4208, found 725.4227.

\section{(+)-TES Ether (60):}

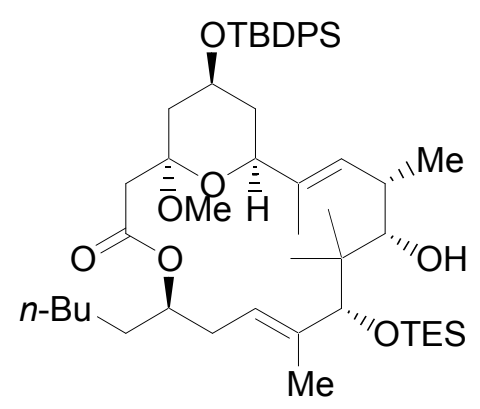

60

43 (6 mg, $8.2 \mu \mathrm{mol})$ was loaded into a $5 \mathrm{~mL}$ RBF and azeotroped with anhydrous toluene, placed under argon and cooled to $-78{ }^{\circ} \mathrm{C}$. Portions $(200 \mu \mathrm{L})$ of a cold $\left(0{ }^{\circ} \mathrm{C}\right)$ stock solution of triethylsilyltriflate (TESOTf) $(70 \mu \mathrm{L}, 0.31 \mathrm{mmol}), 2,6$-lutidine $(71 \mu \mathrm{L}, 0.61 \mathrm{mmol})$ in $\mathrm{CH}_{2} \mathrm{Cl}_{2}(6.0 \mathrm{~mL})$ were added, and the reaction progress checked by TLC (6:1 hexanes:ethyl 
acetate). Additional portions of the stock solution were added until TLC indicated that all the $\mathbf{4 3}$ was consumed. The reaction was quenched with methanol $(70 \mu \mathrm{L})$ diluted with diethyl ether and washed with $0.1 \mathrm{M} \mathrm{NaHSO}_{4}$ and brine, dried over magnesium sulfate, filtered, concentrated and purified by chromatography (hexanes $\rightarrow 13: 1$ hexanes:ethyl acetate) to give $\mathbf{6 0}$ as a clear oil (5.8 mg, 83\%). $[\alpha]_{\mathrm{D}}+34.9\left(c 0.25,22{ }^{\circ} \mathrm{C}, \mathrm{CHCl}_{3}\right)$; IR 3546 (bw), 2955 (s), 2930 (s), 2875 (s), 1728 (s), 1458 (m), 1379 (m), 1238 (m), 1112 (s), 1066 (s), 1045 (s), 1008 (s), 702 (s); ${ }^{1} \mathrm{H}$ NMR (500 $\left.\mathrm{MHz}, \mathrm{CDCl}_{3}\right) \delta 7.68(\mathrm{dd}, J=8.0,1.5 \mathrm{~Hz}, 2 \mathrm{H}), 7.65(\mathrm{dd}, J=8.0,1.5 \mathrm{~Hz}, 2 \mathrm{H}), 7.44(\mathrm{~m}, 2 \mathrm{H}), 7.38$ (m, 4H), $5.80(\mathrm{bs}, 1 \mathrm{H}), 5.60(\mathrm{~d}, J=11.0 \mathrm{~Hz}, 1 \mathrm{H}), 4.88(\mathrm{~m}, 1 \mathrm{H}), 4.20(\mathrm{~s}, 1 \mathrm{H}), 4.14(\mathrm{~m}, 1 \mathrm{H}), 3.64$ $(\mathrm{d}, J=12.0 \mathrm{~Hz}, 1 \mathrm{H}), 3.28(\mathrm{~d}, J=11.0 \mathrm{~Hz}, 1 \mathrm{H}), 2.96(\mathrm{~s}, 3 \mathrm{H}), 2.92(\mathrm{~d}, J=14.5 \mathrm{~Hz}, 1 \mathrm{H}), 2.85(\mathrm{~m}$, 1H), $2.44(\mathrm{~m}, 1 \mathrm{H}), 2.41(\mathrm{~d}, J=14.5 \mathrm{~Hz}, 1 \mathrm{H}), 1.97(\mathrm{~m}, 2 \mathrm{H}), 1.90(\mathrm{~m}, 1 \mathrm{H}), 1.72-1.67$ (bm, 1H), $1.70(\mathrm{~s}, 3 \mathrm{H}), 1.52(\mathrm{~s}, 3 \mathrm{H}), 1.48-1.42(\mathrm{bm}, 1 \mathrm{H}), 1.34-1.24(\mathrm{bm}, 10 \mathrm{H}), 1.05(\mathrm{~s}, 9 \mathrm{H}), 1.02(\mathrm{bs}, 2 \mathrm{H})$, $0.98(\mathrm{~d}, J=6.5 \mathrm{~Hz}, 3 \mathrm{H}), 0.92(\mathrm{t}, J=8.0 \mathrm{~Hz}, 9 \mathrm{H}), 0.92(\mathrm{~d}, J=5.0 \mathrm{~Hz}, 3 \mathrm{H}), 0.88(\mathrm{t}, J=6.5 \mathrm{~Hz}$, 3H), $0.54(\mathrm{q}, J=8.0 \mathrm{~Hz}, 6 \mathrm{H}) ;{ }^{13} \mathrm{C}\left(125.8 \mathrm{MHz}, \mathrm{CDCl}_{3}\right) \delta 169.4,136.0,135.9,134.6,134.5$, 131.0, 129.8, 127.8, 124.1, 99.6, 84.9, 79.8, 74.6, 73.0, 66.8, 48.1, 45.3, 43.4, 42.6, 39.0, 33.9, $33.5,32.0,31.1,29.9,27.1,25.7,22.8,21.5,19.2,14.3,14.1,13.8,7.3,5.2$; HRMS $m / z$ (ESI, $\mathrm{M}+\mathrm{Na}^{+}$) calcd 871.5335, found 871.5358.

\section{(+)-Ketone (61):}

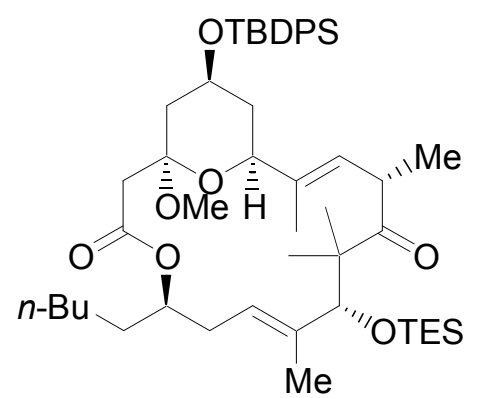

61 
A portion $(3.4 \mathrm{~mL})$ of a stock solution of Dess-Martin periodinane (100 $\mathrm{mg}, 0.23 \mathrm{mmol})$ and pyridine $(93 \mu \mathrm{L}, 1.15 \mathrm{mmol})$ in DCM $(7.14 \mathrm{~mL})$ was added to $60(5.6 \mathrm{mg}, 6.6 \mu \mathrm{mol})$ and the solution was stirred for 20 minutes. The solvent was removed in vacu. and the crude material was purified by silica gel chromatography (hexanes $\rightarrow$ 11:1 hexanes/ethyl acetate) to give $\mathbf{2 6}$ as

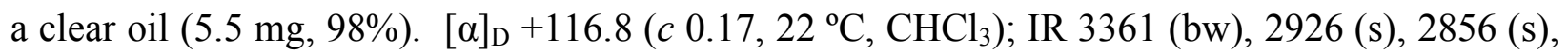
1726 (m), 1709 (w), 1380 (m), 1238 (w), 1111 (s), 1072 (s), 1046 (s), 702 (s); ${ }^{1} \mathrm{H}$ NMR (500 $\left.\mathrm{MHz}, \mathrm{CDCl}_{3}\right) \delta 7.68$ (apparent dd, $\left.J=8.0,1.5 \mathrm{~Hz}, 2 \mathrm{H}\right), 7.65(\mathrm{dd}, J=8.0,1.5 \mathrm{~Hz}, 2 \mathrm{H}), 7.44(\mathrm{~m}$, 2H), $7.38(\mathrm{~m}, 4 \mathrm{H}), 5.40(\mathrm{~d}, J=10.3 \mathrm{~Hz}, 1 \mathrm{H}), 5.25(\mathrm{t}, J=6.6 \mathrm{~Hz}, 1 \mathrm{H}), 4.89(\mathrm{~m}, 1 \mathrm{H}), 4.18(\mathrm{~s}$, $1 \mathrm{H}), 4.10(\mathrm{~m}, 1 \mathrm{H}), 3.97(\mathrm{~m}, 1 \mathrm{H}), 3.68(\mathrm{~d}, J=11.7 \mathrm{~Hz}, 1 \mathrm{H}), 2.94(\mathrm{~s}, 3 \mathrm{H}), 2.88(\mathrm{~d}, J=14.3 \mathrm{~Hz}$, $1 \mathrm{H}), 2.38(\mathrm{~d}, J=14.3 \mathrm{~Hz}, 1 \mathrm{H}), 2.27(\mathrm{~m}, 2 \mathrm{H}), 1.97(\mathrm{ddd}, J=12.8,4.8,1.6 \mathrm{~Hz}, 1 \mathrm{H}), 1.86\left(\mathrm{dt}, J_{\mathrm{t}}=\right.$ $\left.2.2, J_{\mathrm{d}}=12.2 \mathrm{~Hz}, 1 \mathrm{H}\right), 1.78(\mathrm{dd}, J=12.7,10.7 \mathrm{~Hz}, 1 \mathrm{H}), 1.67-1.61(\mathrm{bm}, 1 \mathrm{H}), 1.59(\mathrm{~s}, 3 \mathrm{H}), 1.56$ (s, 3H), 1.43-1.33 (bm, 1H), 1.32-1.22 (bm, 6H), 1.29 (s, 3H), 1.17 (q, $J=11.9 \mathrm{~Hz}, 1 \mathrm{H}), 1.05$ $(\mathrm{s}, 9 \mathrm{H}), 1.01(\mathrm{~d}, J=6.6 \mathrm{~Hz}, 3 \mathrm{H}), 1.00(\mathrm{~s}, 3 \mathrm{H}), 0.97(\mathrm{t}, J=7.8 \mathrm{~Hz}, 9 \mathrm{H}), 0.87(\mathrm{t}, J=7.0 \mathrm{~Hz}, 3 \mathrm{H})$,

$0.60(\mathrm{q}, J=8.0 \mathrm{~Hz}, 6 \mathrm{H}) ;{ }^{13} \mathrm{C}\left(125.8 \mathrm{MHz}, \mathrm{CDCl}_{3}\right) \delta 214,8,169.6,137.8,136.0,135.9,134.6$, $134.4,133.9,129.9,127.8,125.3,123.3,99.5,80.2,74.6,72.5,66.6,56.4,48.4,43.6,42.0,40.6$, $38.9,33.3,32.1,30.8,27.1,25.7,24.4,22.8,19.4,19.2,18.8,14.3,13.8,13.3,7.3,5.0$; HRMS $m / z\left(\mathrm{ESI}, \mathrm{M}+\mathrm{Na}^{+}\right)$calcd 869.5178, found 869.5146.

\section{(+)-(5)-(tert-butyl-diphenyl-silanyloxy)-acutiphycin (49):}




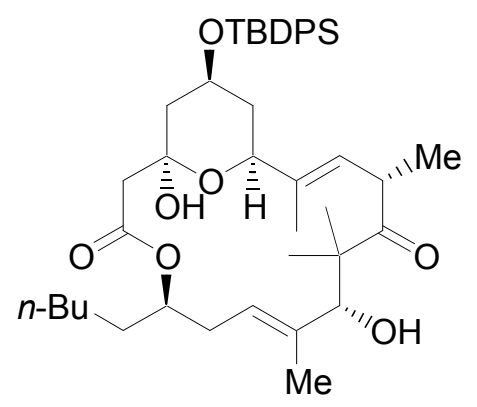

49

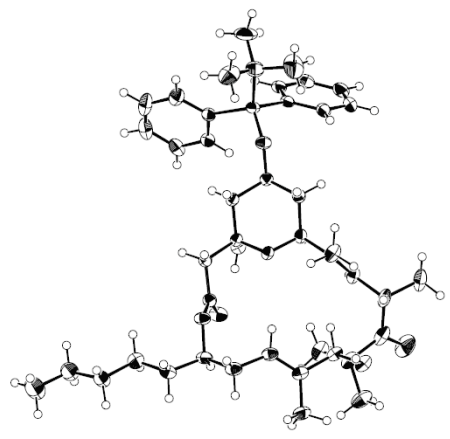

$\mathrm{Et}_{2} \mathrm{O}$ and disorder in $\mathrm{C}_{5} \mathrm{H}_{11}$ chain omitted for clarity

$61(8.3 \mathrm{mg}, 9.8 \mu \mathrm{mol})$ was treated with $49 \%$ aqueous $\mathrm{HF}(74 \mu \mathrm{L}, 2.3 \mathrm{mmol})$ in $\mathrm{MeCN}$ $(667 \mu \mathrm{L})$ at room temperature. After 3 minutes the reaction was poured into a separation funnel containing aqueous sodium bicarbonate and diluted with diethyl ether. The organic layer was washed with sodium bicarbonate and brine, dried over magnesium sulfate, filtered, concentrated, and purified by silica gel chromatography (2:1 hexanes:diethyl ether) to give $\mathbf{4 9}$ as a white solid (6.1 mg, 87\%). The solid was crystallized using slow vapor diffusion of pentanes into diethyl ether (CIF file included in supporting information). $\mathrm{mp} 154-155^{\circ} \mathrm{C} ;[\alpha]_{\mathrm{D}}+131.2\left(c 0.155,22^{\circ} \mathrm{C}\right.$, $\mathrm{CHCl}_{3}$ ); IR 3428 (bs), 2957 (s), 2929 (s), 1703 (s), 1428 (m), 1379 (s), 1210 (m), 1112 (s). ${ }^{1} \mathrm{H}$ NMR (500 MHz, $\left.\mathrm{CDCl}_{3}\right) \delta 7.67$ (apparent d, $\left.J=6.5 \mathrm{~Hz}, 4 \mathrm{H}\right), 7.43(\mathrm{~m}, 2 \mathrm{H}), 7.38(\mathrm{~m}, 4 \mathrm{H}), 5.25$ $(\mathrm{d}, J=9.8 \mathrm{~Hz}, 1 \mathrm{H}), 5.18(\mathrm{dd}, J=11.1,1.1 \mathrm{~Hz}, 1 \mathrm{H}), 5.15(\mathrm{~d}, J=2.6 \mathrm{~Hz}, 1 \mathrm{H}), 4.94(\mathrm{~m}, 1 \mathrm{H}), 4.61$ $(\mathrm{d}, J=4.2 \mathrm{~Hz}, 1 \mathrm{H}), 4.28(\mathrm{~m}, 1 \mathrm{H}), 4.12(\mathrm{dd}, J=11.7,2.2 \mathrm{~Hz}, 1 \mathrm{H}), 3.90(\mathrm{~m}, 1 \mathrm{H}), 2.59(\mathrm{~d}, J=$ 14.6 Hz, 1H), $2.50(\mathrm{~d}, J=14.6 \mathrm{~Hz}, 1 \mathrm{H}), 2.39(\mathrm{ddd}, J=15.2,10.7,2.1 \mathrm{~Hz}, 1 \mathrm{H}), 2.08(\mathrm{t}, J=13.5$ Hz, 1H), 1.99 (dd, $J=12.2,4.5 \mathrm{~Hz}, 1 \mathrm{H}), 1.75$ (d, $J=1.1 \mathrm{~Hz}, 3 \mathrm{H}), 1.73-1.69(\mathrm{~m}, 1 \mathrm{H}), 1.64$ (s, $3 \mathrm{H}), 1.57-1.48(\mathrm{~m}, 3 \mathrm{H}), 1.37\left(\mathrm{dt}, J_{\mathrm{d}}=2.4, J_{\mathrm{t}}=11.5 \mathrm{~Hz}, 1 \mathrm{H}\right), 1.32-1.22(\mathrm{~m}, 7 \mathrm{H}), 1.09(\mathrm{~s}, 3 \mathrm{H})$, $1.07(\mathrm{~s}, 9 \mathrm{H}), 1.02(\mathrm{~d}, J=6.4 \mathrm{~Hz}, 3 \mathrm{H}), 0.87(\mathrm{t}, J=6.9 \mathrm{~Hz}, 3 \mathrm{H}), 0.83(\mathrm{~s}, 3 \mathrm{H}) ;{ }^{13} \mathrm{C}(125.8 \mathrm{MHz}$, $\left.\mathrm{CDCl}_{3}\right) \delta 215.7,172.6,135.9,135.3,134.9,134.4,134.4,130.7,129.9,129.9,127.8,126.6,96.8$ $79.9,75.9,74.2,66.3,52.8,44.8,44.2,43.3,38.5,35.5,32.9,31.8,27.2,25.8,25.2,22.7,19.4$, 19.3, 16.2, 14.2, 13.1, 11.3; HRMS $m / z\left(E S I, M+\mathrm{Na}^{+}\right)$calcd 741.4157, found 741.4150. 


\section{(+)-Acutiphycin (1):}

For comparison the chemical shifts for (1) as reported by Moore are listed below. ${ }^{8}$

${ }^{1} \mathrm{H}$ NMR $\left(300 \mathrm{MHz}, 1: 1 \mathrm{C}_{6} \mathrm{D}_{6}: \mathrm{CDCl}_{3}\right.$ ) (carbon on which the protons are located given in parenthesis) $\delta 5.36$ (OH on C3), 5.20 (C15), 5.15 (C9), 4.89 (C17), 4.51 (C13), 4.16 (C7), 4.0

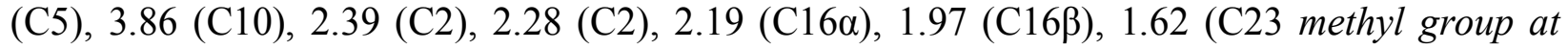
C8), 1.54 (C6), 1.43 (C27 methyl group at C14), 1.30 (C6), 1.16 (C18, C19, C20, C21), 1.09 (C26 methyl group at C12), 1.03 (C24 methyl group at C10), 0.99 (C4), 0.84 (C25 methyl group at C12), $0.80(\mathrm{C} 22) ;{ }^{13} \mathrm{C}\left(75 \mathrm{MHz}, \mathrm{Me}_{2} \mathrm{SO}-d_{6}\right) \delta 124.4$ (likely a typo and was meant to be 214.4 as observed by ourselves and by Smith) (C11), 170.9 (C1), 136.1 (C8 or C14), 134.9 (C8 or C14), 128.1 (C9), 123.6 (C15), 96.2 (C3), 77.3 (C13), 74.2 (C7), 73.8 (C17), 62.6 (C5), 53.0 (C12), 49.9 (C2), 43.5 (C4), 41.3 (C10), 38.2 (C6), 34.4 (C16), 31.8 (C20), 31.0 (C18), 24.4 (C19), 23.4 (C26 methyl group at C12), 22.0 (C21), 20.6 (C25 methyl group at C12), 16.7 (C24 methyl group at C10), 13.9 (C22), 12.9 (C27 methyl group at C14), 11.9 (C23 methyl group at C8). 
Figure 1: (+)-acutiphycin (1) in $\mathrm{CDCl}_{3}$

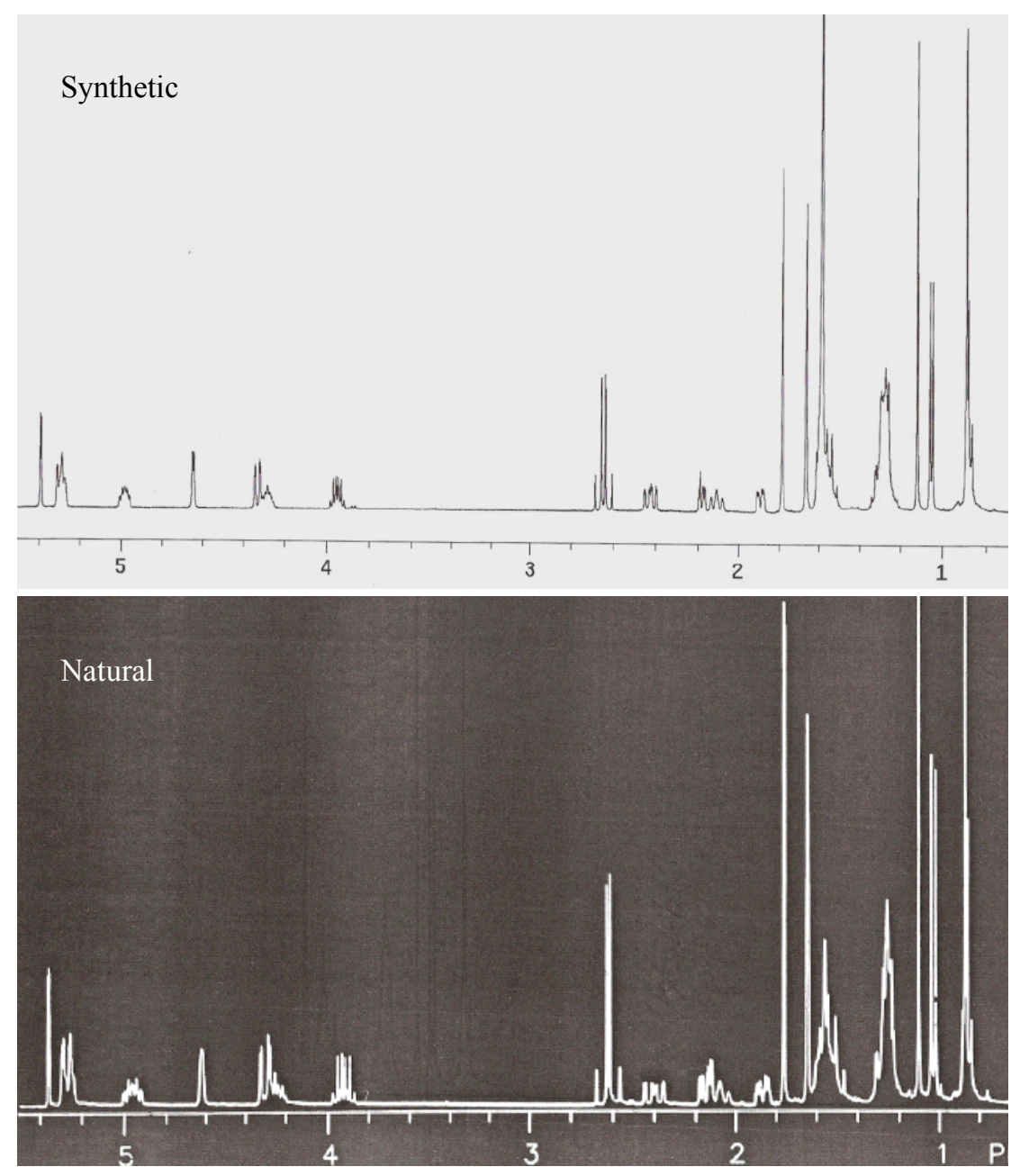




\section{References}

(1) Eggen, M.; Mossman, C. J.; Buck, S. B.; Nair, S. K.; Bhat, L.; Ali, S. M.; Reiff, E. A.; Boge, T. C.; Georg, G. I. J. Org. Chem. 2000, 65, 7792-7799.

(2) Roush, W. R.; Palkowitz, A. D.; Ando, K. J. Am. Chem. Soc. 1990, 112, 6348-6359.

(3) (a) Tokunaga, M.; Larrow, J. F.; Kakuchi, F.; Jacobsen, E. N. Science, 1997, 277, 936-938. (b) Schaus, S. E.; Brandes, B. D.; Larrow, J. F.; Tokunaga, M.; Hansen, K. B.; Gould, A. E.; Furrow, M. E.; Jacobsen, E. N. J. Am. Chem. Soc. 2002, 124, 1307-1315.

(4) Gupta, P. S.; Naidu, V.; Kumar, P. Tetrahedron Lett. 2004, 45, 849-851.

(5) Available in one step from tiglic acid: Aurell, M. J.; Gil, S.; Mestres, R.; Parra, M.; Parra, L. Tetrahedron 1998, 54, 4357-4366.

(6) Marshall, J. A.; Adams, N. D. J. Org. Chem. 2002, 67, 733-740.

(7) Shioji, K.; Kawaoka, H.; Miura, A.; Okuma, K. Synth. Commun. 2001, 31, 3569-3575.

(8) Barchi, J. J., Jr.; Moore, R. E.; Patterson, F. M. L. J. Am. Chem. Soc. 1984, 106, 8193-8197. 